TRANSACTIONS OF THE

AMERICAN MATHEMATICAL SOCIETY

Volume 356, Number 1, Pages 135-157

S 0002-9947(03)03028-9

Article electronically published on August 21, 2003

\title{
GEOMETRIC ASPECTS OF STURM-LIOUVILLE PROBLEMS II. SPACE OF BOUNDARY CONDITIONS FOR LEFT-DEFINITENESS
}

\author{
KEVIN HAERTZEN, QINGKAI KONG, HONGYOU WU, AND ANTON ZETTL
}

\begin{abstract}
For a given regular Sturm-Liouville equation with an indefinite weight function, we explicitly describe the space of left-definite selfadjoint boundary conditions. The description only uses one value of a fundamental solution of the matrix form of the equation. As a consequence we show that this space has the shape of a solid consisting of two cones sharing a common base.
\end{abstract}

We continue to study spectral problems associated with a regular Sturm-Liouville equation (SLE), i.e.,

$$
-\left(p y^{\prime}\right)^{\prime}+q y=\lambda w y \text { on }(a, b),
$$

where $\lambda \in \mathbb{C}$ is the so-called spectral parameter,

$$
-\infty \leq a<b \leq+\infty, \quad 1 / p, q, w \in \mathrm{L}((a, b), \mathbb{R}) .
$$

Here $\mathrm{L}((a, b), \mathbb{R})$ denotes the space of Lebesgue integrable real-valued functions on $(a, b)$.

In this paper, we consider the indefinite case where

$$
\begin{gathered}
p>0 \text { a.e. on }(a, b), \quad w \neq 0 \text { a.e. on }(a, b), \\
\mathrm{m}(\{t \in(a, b) ; w(t)>0\})>0, \quad \operatorname{m}(\{t \in(a, b) ; w(t)<0\})>0,
\end{gathered}
$$

where for a Lebesgue measurable set $S \subseteq \mathbb{R}, \mathrm{m}(S)$ is the measure of $S$. The weight function $w$ is said to be indefinite when $(0.4)$ holds, and definite when it is either positive a.e. on $(a, b)$ or negative a.e. on $(a, b)$. Under the assumptions $(0.3)$ and (0.4), the Sturm-Liouville problem (SLP) consisting of (0.1) and a selfadjoint boundary condition (BC) may have non-real eigenvalues (see, for example, [6] and the references therein). A major question about such a problem, i.e., how many non-real eigenvalues it has, is still open. Nevertheless, there is a subclass of such problems, the so-called left-definite (LD) ones, which have only real eigenvalues. A characterization of the LD problems is well known (see, for example, [5]). To state it, we also consider the associated right-definite (RD) equation

$$
-\left(p y^{\prime}\right)^{\prime}+q y=\zeta|w| y \text { on }(a, b)
$$

Received by the editors November 16, 2001.

2000 Mathematics Subject Classification. Primary 34B24, 34B09; Secondary 34L05, 34L15.

Key words and phrases. Regular Sturm-Liouville problems, left-definiteness, spaces of boundary conditions. 
of (0.1). Note that $\zeta$ is used in (0.5) as the spectral parameter. The existing characterization says that the SLP consisting of (0.1) and a selfadjoint BC is LD if and only if the SLP consisting of the associated RD equation (0.5) and the same $\mathrm{BC}$ has only positive eigenvalues, i.e., if and only if the first eigenvalue of the latter problem is positive. Since the first Dirichlet eigenvalue $\zeta_{0}^{D}$ is the maximum of the first eigenvalue $\zeta_{0}$ of $(0.5)$ on the space of selfadjoint BC's 44, the above characterization implies that the space of LD selfadjoint BC's is non-empty if and only if $\zeta_{0}^{D}>0$. However, this characterization does not give a description of the space of LD selfadjoint BC's.

In this paper, we give an explicit description of the space of $\mathrm{LD}$ selfadjoint $\mathrm{BC}$ 's. The same is achieved for the space of semi-left-definite (SLD) selfadjoint BC's. Both descriptions only use one value of a fundamental solution of the matrix form of $(0.1)$.

The main ideas in this work can be explained as follows. By the known characterization, the space $\mathcal{B}_{\mathrm{L}}^{\mathbb{C}}$ of LD selfadjoint BC's is the subset of the space $\mathcal{B}^{\mathbb{C}}$ of selfadjoint BC's where $\zeta_{0}>0$. If $\zeta_{0}$ were continuous on $\mathcal{B}^{\mathbb{C}}$, then $\mathcal{B}_{\mathrm{L}}^{\mathbb{C}}$ would be bounded by (part of) the zero set of $\zeta_{0}$. However, $\zeta_{0}$ has a non-empty discontinuity set, to be called the jump set: $\zeta_{0}$ always jumps to $-\infty$ on one side of this set [4]. The jump set contains the Dirichlet BC. Thus, $\mathcal{B}_{\mathrm{L}}^{\mathbb{C}}$ is bounded by (part of) both the zero set and the jump set. These two sets are determined in 3$]$ and [4]. Let $\mathcal{P}^{\mathbb{C}}$ be the union of these two sets. Then, on each connected component of the complement of $\mathcal{P}^{\mathbb{C}}$ in $\mathcal{B}^{\mathbb{C}}, \zeta_{0}$ does not change sign. The signs of $\zeta_{0}$ on these components can be determined in terms of some monotonicity results about $\zeta_{0}$ on the space of separated selfadjoint BC's in 2] and some geometric coordinate transformations on $\mathcal{B}^{\mathbb{C}}$ introduced in $[3]$. We thus see which components are in $\mathcal{B}_{\mathrm{L}}^{\mathbb{C}}$ and hence obtain a description of $\mathcal{B}_{\mathrm{L}}^{\mathbb{C}}$. Let $\Phi$ be the fundamental solution of the matrix form of (0.1) satisfying $\Phi(a, \cdot)=I$, and set $C=\Phi(b, 0)$. Note that one gets the same matrix $C$ if (0.5) is used instead of (0.1). From [3] and [4] we know that the zero set only depends on $C$ and the jump set is independent of the differential equation (0.5). So, our description of $\mathcal{B}_{\mathrm{L}}^{\mathbb{C}}$ only uses the constant matrix $C$.

As a by-product of our description of the space of SLD selfadjoint BC's, for a given SLE with a positive leading coefficient and a definite weight function, we obtain the collection of selfadjoint BC's whose first eigenvalue is equal to the first Dirichlet eigenvalue. Our approach here can be modified to yield the level surfaces of the $n$-th eigenvalue of such an equation for each $n \in \mathbb{N}$. We will do this in the next paper of this series.

We also show that $\mathcal{B}_{\mathrm{L}}^{\mathbb{C}}$ is diffeomorphic to a solid consisting of two cones sharing a common base. Half of the boundary of this solid does not belong to $\mathcal{B}_{\mathrm{L}}^{\mathbb{C}}$, while the space $\mathcal{B}_{\mathrm{SL}}^{\mathbb{C}}$ of SLD selfadjoint BC's is the closure of $\mathcal{B}_{\mathrm{L}}^{\mathbb{C}}$. When $\mathcal{B}_{\mathrm{L}}^{\mathbb{C}}$ is empty and $\mathcal{B}_{\mathrm{SL}}^{\mathbb{C}}$ is not, $\mathcal{B}_{\mathrm{SL}}^{\mathbb{C}}$ is a simple smooth curve joining the Dirichlet $\mathrm{BC}$ to the unique coupled $\mathrm{BC}$ having 0 as a double eigenvalue.

For each $r \in \mathbb{R}$, the concepts of so-called $r$-left-definiteness and $r$-semi-leftdefiniteness were introduced in [5]. Our results here easily extend to cover this more general case. We omit the details.

The organization of this paper is as follows. In Section 1, we introduce our notation, recall some basic results and prove a couple of lemmas. To better illustrate the main ideas of our approach and also prepare for dealing with general selfadjoint BC's, we first single out in Section 2 the separated LD selfadjoint BC's. Section 3 is devoted to determining the real LD selfadjoint BC's, while Section 4 contains 
the complex case. In Section 5, we then find the geometric shapes of the spaces of real and complex LD selfadjoint BC's.

\section{$\S 1$. Notation AND BASIC RESUlts}

In this section, in addition to defining our notation, we work out some decompositions of the space of selfadjoint BC's, summarize some basic results about the LD problems and recall some facts about the eigenvalues of (0.5).

For any $m, n \in \mathbb{N}$, we use $\mathrm{M}_{m, n}(\mathbb{C})$ to denote the vector space of $m$ by $n$ complex matrices and $\mathrm{M}_{m, n}^{*}(\mathbb{C})$ its open subspace consisting of the elements with the maximum rank $\min \{m, n\}$, while $\mathrm{M}_{m, n}(\mathbb{R})$ and $\mathrm{M}_{m, n}^{*}(\mathbb{R})$ are the real analogs of $\mathrm{M}_{m, n}(\mathbb{C})$ and $\mathrm{M}_{m, n}^{*}(\mathbb{C})$, respectively. Let $\mathrm{GL}(2, \mathbb{C})$ be the Lie group of invertible complex matrices in dimension 2 and $\mathrm{SL}(2, \mathbb{R})$ its subgroup consisting of the real elements having determinant 1 . When a capital Latin or Greek letter stands for a matrix, the entries of the matrix will always be denoted by the corresponding lower case letter with two indices.

Following [3], we will take the quotient space

$$
\mathrm{GL}(2, \mathbb{C}) \backslash \mathrm{M}_{2,4}^{*}(\mathbb{C})
$$

as the space of BC's, i.e., each $B C$ is an equivalence class of coefficient matrices (with the elements of $\mathrm{GL}(2, \mathbb{C})$ multiplying from the left) of linear systems

$$
(A \mid B)\left(\begin{array}{c}
y(a) \\
\left(p y^{\prime}\right)(a) \\
y(b) \\
\left(p y^{\prime}\right)(b)
\end{array}\right)=0
$$

with $\operatorname{rank}(A \mid B)=2$, and the $\mathrm{BC}$ represented by the linear system (1.2) will be denoted by $[A \mid B]$. Note here that square brackets, not parentheses, are used. Usual bold faced capital Latin letters, such as $\boldsymbol{A}$, will also be used for BC's.

Motivated by the right-definite theory, a $\mathrm{BC}[A \mid B]$ is said to be selfadjoint if

$$
A\left(\begin{array}{cc}
0 & 1 \\
-1 & 0
\end{array}\right) A^{*}=B\left(\begin{array}{cc}
0 & 1 \\
-1 & 0
\end{array}\right) B^{*}
$$

where $A^{*}$ is the complex conjugate transpose of $A$. The space $\mathcal{B}^{\mathbb{R}}$ of real selfadjoint BC's consists of the separated real BC's and the coupled real BC's of the form $[K \mid-I]$ with $K \in \mathrm{SL}(2, \mathbb{R})$. By Theorem 3.9 in $\left[3, \mathcal{B}^{\mathbb{R}}\right.$ is a connected and compact analytic 3-dimensional manifold. It can be obtained by "gluing" the open sets

$$
\begin{aligned}
& \mathcal{O}_{1}^{\mathbb{R}}=\mathcal{O}_{6}^{\mathbb{R}}=\{[K \mid-I] ; K \in \operatorname{SL}(2, \mathbb{R})\}, \\
& \mathcal{O}_{2}^{\mathbb{R}}=\left\{\left[\begin{array}{cccc}
1 & a_{12} & 0 & a_{22} \\
0 & a_{22} & -1 & b_{22}
\end{array}\right] ; a_{12}, a_{22}, b_{22} \in \mathbb{R}\right\}, \\
& \mathcal{O}_{3}^{\mathbb{R}}=\left\{\left[\begin{array}{cccc}
1 & a_{12} & -a_{22} & 0 \\
0 & a_{22} & b_{21} & -1
\end{array}\right] ; a_{12}, a_{22}, b_{21} \in \mathbb{R}\right\}, \\
& \mathcal{O}_{4}^{\mathbb{R}}=\left\{\left[\begin{array}{cccc}
a_{11} & 1 & 0 & -a_{21} \\
a_{21} & 0 & -1 & b_{22}
\end{array}\right] ; a_{11}, a_{21}, b_{22} \in \mathbb{R}\right\}, \\
& \mathcal{O}_{5}^{\mathbb{R}}=\left\{\left[\begin{array}{cccc}
a_{11} & 1 & a_{21} & 0 \\
a_{21} & 0 & b_{21} & -1
\end{array}\right] ; a_{11}, a_{21}, b_{21} \in \mathbb{R}\right\}
\end{aligned}
$$

via the coordinate transformations among these open sets. Note that the topology on $\operatorname{SL}(2, \mathbb{R})$ is the one induced from the usual topology on the space $\mathrm{M}_{2,2}(\mathbb{R})$, and 
each of the four open sets in (1.5)-(1.8) can be identified with $\mathbb{R}^{3}$. A $\mathrm{BC}[A \mid B]$ is selfadjoint if and only if either it is a real selfadjoint $\mathrm{BC}$ or $[A \mid B]=\left[\mathrm{e}^{\mathrm{i} \theta} K \mid-I\right]$ with $\theta \in(0, \pi)$ and $K \in \mathrm{SL}(2, \mathbb{R})$. By Theorem 3.11 in [3], the space $\mathcal{B}^{\mathbb{C}}$ of selfadjoint BC's is a connected and compact analytic 4-dimensional real manifold. It can be obtained by "gluing" the open sets

$$
\begin{aligned}
& \mathcal{O}_{1}^{\mathbb{C}}=\mathcal{O}_{6}^{\mathbb{C}}=\left\{\left[\mathrm{e}^{\mathrm{i} \theta} K \mid-I\right] ; \theta \in[0, \pi), K \in \mathrm{SL}(2, \mathbb{R})\right\}, \\
& \mathcal{O}_{2}^{\mathbb{C}}=\left\{\left[\begin{array}{cccc}
1 & a_{12} & 0 & \bar{z} \\
0 & z & -1 & b_{22}
\end{array}\right] ; a_{12} \in \mathbb{R}, z \in \mathbb{C}, b_{22} \in \mathbb{R}\right\} \text {, } \\
& \mathcal{O}_{3}^{\mathbb{C}}=\left\{\left[\begin{array}{cccc}
1 & a_{12} & -\bar{z} & 0 \\
0 & z & b_{21} & -1
\end{array}\right] ; a_{12} \in \mathbb{R}, z \in \mathbb{C}, b_{21} \in \mathbb{R}\right\}, \\
& \mathcal{O}_{4}^{\mathbb{C}}=\left\{\left[\begin{array}{cccc}
a_{11} & 1 & 0 & -\bar{z} \\
z & 0 & -1 & b_{22}
\end{array}\right] ; a_{11} \in \mathbb{R}, z \in \mathbb{C}, b_{22} \in \mathbb{R}\right\}, \\
& \mathcal{O}_{5}^{\mathbb{C}}=\left\{\left[\begin{array}{cccc}
a_{11} & 1 & \bar{z} & 0 \\
z & 0 & b_{21} & -1
\end{array}\right] ; a_{11} \in \mathbb{R}, z \in \mathbb{C}, b_{21} \in \mathbb{R}\right\}
\end{aligned}
$$

via the coordinate transformations among these open sets. Note that the topology on the open set in (1.9) is the one induced from the usual topology on the space $\mathrm{M}_{2,2}(\mathbb{C})$, and each of the four open sets in (1.10)-(1.13) can be identified with $\mathbb{R}^{4}$.

The Lie group $\operatorname{SL}(2, \mathbb{R})$ acts on $\mathcal{B}^{\mathbb{R}}$ and $\mathcal{B}^{\mathbb{C}}$ from the right (see $\S 3$ of $[3]$ ) and the actions are given by

$$
[A \mid B] \cdot K=[A K \mid B]
$$

for $[A \mid B] \in \mathcal{B}^{\mathbb{C}}$ and $K \in \mathrm{SL}(2, \mathbb{R})$.

Let $\boldsymbol{D}$ be the Dirichlet BC, DN the Dirichlet-Neumann BC, ND the NeumannDirichlet BC, $\boldsymbol{N}$ the Neumann BC, and set

$$
\begin{aligned}
\mathcal{K}^{\mathbb{R}} & =\left\{\left[\begin{array}{cccc}
1 & a_{2} & 0 & r \\
0 & r & -1 & b_{2}
\end{array}\right] ; \quad a_{2}, b_{2}, r \in \mathbb{R}, a_{2} b_{2}=r^{2}\right\}, \\
\mathcal{M}^{\mathbb{R}} & =\left\{\left[\begin{array}{cccc}
r & 0 & -1 & 0 \\
0 & 1 / r & 0 & -1
\end{array}\right] ; \quad r \in \mathbb{R}^{*}\right\} \cup\{\mathrm{DN}, \mathrm{ND}\}, \\
\mathcal{K}^{\mathbb{C}} & =\left\{\left[\begin{array}{cccc}
1 & a_{2} & 0 & \bar{z} \\
0 & z & -1 & b_{2}
\end{array}\right] ; \quad a_{2}, b_{2} \in \mathbb{R}, z \in \mathbb{C}, a_{2} b_{2}=z \bar{z}\right\}, \\
\mathcal{M}^{\mathbb{C}} & =\left\{\left[\begin{array}{cccc}
\mathrm{e}^{\mathrm{i} \theta} r & 0 & -1 & 0 \\
0 & \mathrm{e}^{\mathrm{i} \theta} / r & 0 & -1
\end{array}\right] ; \quad \begin{array}{c}
r \in \mathbb{R}^{*} \\
\end{array}\right\} \cup\{\mathrm{DN}, \mathrm{ND}\},
\end{aligned}
$$

where $\mathbb{R}^{*}=\mathbb{R} \backslash\{0\}$. Note that $\mathcal{K}^{\mathbb{R}}$ is a cone in $\mathcal{O}_{2}^{\mathbb{R}} \simeq \mathbb{R}^{3}$ and $\mathcal{M}^{\mathbb{R}}$ is a circle in $\mathcal{B}^{\mathbb{R}}$, while $\mathcal{K}^{\mathbb{C}}$ is a cone in $\mathcal{O}_{2}^{\mathbb{C}} \simeq \mathbb{R}^{4}$ and $\mathcal{M}^{\mathbb{C}}$ is a sphere in $\mathcal{B}^{\mathbb{C}}$.

Lemma 1.19. For any $K \in \mathrm{SL}(2, \mathbb{R})$, we have that

$$
\begin{gathered}
\mathcal{B}^{\mathbb{R}}=\mathcal{O}_{5}^{\mathbb{R}} \cup \mathcal{K}^{\mathbb{R}} \cup \mathcal{M}^{\mathbb{R}}=\left(\mathcal{O}_{5}^{\mathbb{R}} \bullet K\right) \cup\left(\mathcal{K}^{\mathbb{R}} \bullet K\right) \cup\left(\mathcal{M}^{\mathbb{R}} \bullet K\right), \\
\overline{\mathcal{K}^{\mathbb{R}}}=\mathcal{K}^{\mathbb{R}} \cup \mathcal{M}^{\mathbb{R}}, \quad \overline{\mathcal{K}^{\mathbb{R}} \cdot K}=\left(\mathcal{K}^{\mathbb{R}} \cdot K\right) \cup\left(\mathcal{M}^{\mathbb{R}} \cdot K\right), \overline{\mathcal{O}_{5}^{\mathbb{R}}}=\overline{\mathcal{O}_{5}^{\mathbb{R}} \cdot K}=\mathcal{B}^{\mathbb{R}}, \\
\mathcal{B}^{\mathbb{C}}=\mathcal{O}_{5}^{\mathbb{C}} \cup \mathcal{K}^{\mathbb{C}} \cup \mathcal{M}^{\mathbb{C}}=\left(\mathcal{O}_{5}^{\mathbb{C}} \bullet K\right) \cup\left(\mathcal{K}^{\mathbb{C}} \bullet K\right) \cup\left(\mathcal{M}^{\mathbb{C}} \cdot K\right), \\
\overline{\mathcal{K}^{\mathbb{C}}}=\mathcal{K}^{\mathbb{C}} \cup \mathcal{M}^{\mathbb{C}}, \quad \overline{\mathcal{K}^{\mathbb{C}} \cdot K}=\left(\mathcal{K}^{\mathbb{C}} \cdot K\right) \cup\left(\mathcal{M}^{\mathbb{C}} \cdot K\right), \quad \overline{\mathcal{O}_{5}^{\mathbb{C}}}=\overline{\mathcal{O}_{5}^{\mathbb{C}} \cdot K}=\mathcal{B}^{\mathbb{C}} .
\end{gathered}
$$

All these unions are disjoint. 
Proof. Since a BC

$$
\left[\begin{array}{cccc}
1 & a_{2} & 0 & r \\
0 & r & -1 & b_{2}
\end{array}\right] \in \mathcal{O}_{2}^{\mathbb{R}}
$$

is not in $\mathcal{O}_{5}^{\mathbb{R}}$ if and only if

$$
a_{2} b_{2}-r^{2}=\left|\begin{array}{cc}
a_{2} & r \\
r & b_{2}
\end{array}\right|=0
$$

we have that

$$
\mathcal{O}_{2}^{\mathbb{R}} \backslash \mathcal{O}_{5}^{\mathbb{R}}=\mathcal{K}^{\mathbb{R}}
$$

Similarly,

$$
\begin{aligned}
& \mathcal{O}_{6}^{\mathbb{R}} \backslash \mathcal{O}_{5}^{\mathbb{R}} \backslash \mathcal{O}_{2}^{\mathbb{R}}=\left\{\left[\begin{array}{cccc}
r & 0 & -1 & 0 \\
0 & 1 / r & 0 & -1
\end{array}\right] ; \quad r \in \mathbb{R}^{*}\right\}, \\
& \mathcal{O}_{3}^{\mathbb{R}} \backslash \mathcal{O}_{5}^{\mathbb{R}} \backslash \mathcal{O}_{2}^{\mathbb{R}} \backslash \mathcal{O}_{6}^{\mathbb{R}}=\{\mathrm{DN}\}, \\
& \mathcal{O}_{4}^{\mathbb{R}} \backslash \mathcal{O}_{5}^{\mathbb{R}} \backslash \mathcal{O}_{2}^{\mathbb{R}} \backslash \mathcal{O}_{6}^{\mathbb{R}} \backslash \mathcal{O}_{3}^{\mathbb{R}}=\{\mathrm{ND}\} .
\end{aligned}
$$

Therefore,

$$
\mathcal{B}^{\mathbb{R}}=\mathcal{O}_{5}^{\mathbb{R}} \cup \mathcal{O}_{2}^{\mathbb{R}} \cup \mathcal{O}_{6}^{\mathbb{R}} \cup \mathcal{O}_{3}^{\mathbb{R}} \cup \mathcal{O}_{4}^{\mathbb{R}}=\mathcal{O}_{5}^{\mathbb{R}} \cup \mathcal{K}^{\mathbb{R}} \cup \mathcal{M}^{\mathbb{R}}
$$

with $\mathcal{O}_{5}^{\mathbb{R}}, \mathcal{K}^{\mathbb{R}}$ and $\mathcal{M}^{\mathbb{R}}$ mutually disjoint. Since the action of $K$ on $\mathcal{B}^{\mathbb{R}}$ is a diffeomorphism of $\mathcal{B}^{\mathbb{R}}$ onto itself, (1.30) implies that

$$
\mathcal{B}^{\mathbb{R}}=\left(\mathcal{O}_{5}^{\mathbb{R}} \bullet K\right) \cup\left(\mathcal{K}^{\mathbb{R}} \bullet K\right) \cup\left(\mathcal{M}^{\mathbb{R}} \cdot K\right),
$$

a disjoint union. This completes the proof of (1.20). Similarly, one proves (1.22).

Since $\mathcal{K}^{\mathbb{R}}$ and $\mathcal{M}^{\mathbb{R}}$ have dimensions less than that of $\mathcal{B}^{\mathbb{R}}$, (1.30) yields that $\overline{\mathcal{O}_{5}^{\mathbb{R}}}=\mathcal{B}^{\mathbb{R}}$. Similarly, (1.31) implies that $\overline{\mathcal{O}_{5}^{\mathbb{R}} \cdot K}=\mathcal{B}^{\mathbb{R}}$. Since $\mathcal{O}_{5}^{\mathbb{R}}$ is open, from (1.30) one deduces that $\overline{\mathcal{K}^{\mathbb{R}}} \subseteq \mathcal{K}^{\mathbb{R}} \cup \mathcal{M}^{\mathbb{R}}$. For any $r \in \mathbb{R}^{*}$, from

$$
\left[\begin{array}{cccc}
r & 0 & -1 & 0 \\
\epsilon & 1 / r & 0 & -1
\end{array}\right] \in \mathcal{O}_{2}^{\mathbb{R}} \backslash \mathcal{O}_{5}^{\mathbb{R}}=\mathcal{K}^{\mathbb{R}} \text { for } \epsilon>0
$$

we obtain

$$
\left[\begin{array}{cccc}
r & 0 & -1 & 0 \\
0 & 1 / r & 0 & -1
\end{array}\right] \in \overline{\mathcal{K}^{\mathbb{R}}}
$$

Thus,

$$
\left\{\left[\begin{array}{cccc}
r & 0 & -1 & 0 \\
0 & 1 / r & 0 & -1
\end{array}\right] ; \quad r \in \mathbb{R}^{*}\right\} \subset \overline{\mathcal{K}^{\mathbb{R}}},
$$

which also implies that $\{\mathrm{DN}, \mathrm{ND}\} \subset \overline{\mathcal{K}^{\mathbb{R}}}$. Therefore,

$$
\overline{\mathcal{K}^{\mathbb{R}}}=\mathcal{K}^{\mathbb{R}} \cup \mathcal{M}^{\mathbb{R}},
$$

and hence

$$
\overline{\mathcal{K}^{\mathbb{R}} \bullet K}=\left(\mathcal{K}^{\mathbb{R}} \bullet K\right) \cup\left(\mathcal{M}^{\mathbb{R}} \bullet K\right) .
$$

This finishes the proof of (1.21), and (1.23) can be shown in the same way. 
For each $\lambda \in \mathbb{C}$, let $\phi_{11}(\cdot, \lambda)$ and $\phi_{12}(\cdot, \lambda)$ be the solutions of $(0.1)$ determined by the initial conditions

$$
\phi_{11}(a, \lambda)=1,\left(p \phi_{11}^{\prime}\right)(a, \lambda)=0, \quad \phi_{12}(a, \lambda)=0,\left(p \phi_{12}^{\prime}\right)(a, \lambda)=1 .
$$

We will denote $p \phi_{11}^{\prime}$ and $p \phi_{12}^{\prime}$ by $\phi_{21}$ and $\phi_{22}$, respectively. Set

$$
\Phi(t, \lambda)=\left(\begin{array}{ll}
\phi_{11}(t, \lambda) & \phi_{12}(t, \lambda) \\
\phi_{21}(t, \lambda) & \phi_{22}(t, \lambda)
\end{array}\right), \quad t \in[a, b], \lambda \in \mathbb{C} .
$$

Then, $\Phi(t, \lambda)$ satisfies the matrix form of $(0.1)$, i.e.,

$$
\Phi^{\prime}(t, \lambda)=\left(\begin{array}{cc}
0 & 1 / p(t) \\
q(t)-\lambda w(t) & 0
\end{array}\right) \Phi(t, \lambda) \quad \text { on }(a, b),
$$

and $\Phi(a, \cdot)=I$. We will call $\Phi$ the principal matrix of (0.1). Note that we can define the principal matrix for any regular SLE. In particular, we will do this for (0.5) later.

Now, we introduce the basic concepts of left-definiteness and semi-left-definiteness. For more details, see [5]. We will abbreviate the space $\operatorname{AC}_{\text {loc }}((a, b), \mathbb{C})$ of functions that are absolutely continuous on all compact subintervals of $(a, b)$ as $\mathrm{AC}_{\mathrm{loc}}$.

Definition 1.40. For the SLP consisting of $(0.1)$ and a $\mathrm{BC}[A \mid B] \in \mathcal{B}^{\mathbb{C}}$, define two subspaces of the weighted Hilbert space $\mathrm{L}^{2}=\mathrm{L}^{2}((a, b), \mathbb{C} ;|w|)$ by

$$
\begin{aligned}
& \Gamma_{\max }=\left\{f \in \mathrm{L}^{2} ; \quad f, p f^{\prime} \in \mathrm{AC}_{\mathrm{loc}},\left[-\left(p f^{\prime}\right)^{\prime}+q f\right] /|w| \in \mathrm{L}^{2}\right\}, \\
& \Gamma=\left\{f \in \Gamma_{\max } ; \quad A\left(\begin{array}{c}
f(a) \\
\left(p f^{\prime}\right)(a)
\end{array}\right)+B\left(\begin{array}{c}
f(b) \\
\left(p f^{\prime}\right)(b)
\end{array}\right)=0\right\},
\end{aligned}
$$

and a functional $\tau$ on $\Gamma$ by

$$
\tau f=\int_{a}^{b}\left[-\left(p f^{\prime}\right)^{\prime} \bar{f}+q|f|^{2}\right]
$$

Then, the problem is said to be left-definite (LD) if $\tau$ is definite on $\Gamma$, i.e., either $\tau f>0$ for all $f \not \equiv 0$ in $\Gamma$ or $\tau f<0$ for all $f \not \equiv 0$ in $\Gamma$; it is said to be semi-leftdefinite (SLD) if $\tau$ is semi-definite on $\Gamma$, i.e., either $\tau f \geq 0$ for all $f \in \Gamma$ or $\tau f \leq 0$ for all $f \in \Gamma$.

The following characterization of left-definiteness has been mentioned in the introduction and is taken from Theorem 2.1 in [5]. The characterization of semileft-definiteness is very similar.

Theorem 1.44. The following three statements are equivalent to each other.

i) The Sturm-Liouville problem consisting of (0.1) and a selfadjoint boundary condition is left-definite.

ii) The functional $\tau$ is positive definite on $\Gamma$.

iii) The eigenvalues of the right-definite problem consisting of (0.5) and the same boundary condition are all positive.

The following three statements are also equivalent to each other.

$\left.\mathrm{i}^{\prime}\right)$ The Sturm-Liouville problem consisting of (0.1) and a selfadjoint boundary condition is semi-left-definite.

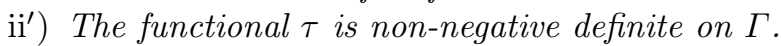

iii') The eigenvalues of the right-definite problem consisting of (0.5) and the same boundary condition are all non-negative. 
Remark 1.45. By Proposition 2.1 in [5], for Theorem 1.44 to hold, the function $|w|$ in (0.5) can be replaced by any positive and integrable function on $(a, b)$.

Proposition 1.46. i) There is a left-definite selfadjoint boundary condition for (0.1) if and only if the Dirichlet boundary condition is such a boundary condition, i.e., if and only if the lowest Dirichlet eigenvalue of (0.5) is positive.

ii) There is a semi-left-definite selfadjoint boundary condition for (0.1) and no left-definite selfadjoint boundary condition for (0.1) if and only if the Dirichlet boundary condition is semi-left-definite for (0.1) but not left-definite for (0.1), i.e., if and only if the lowest Dirichlet eigenvalue of (0.5) equals 0.

Proof. These two facts are direct consequences of Theorem 1.44 above and Theorem 4.1 in [4]: the first eigenvalue of (0.5) takes its maximum at $\boldsymbol{D}$.

The following result, also mentioned in the introduction, shows the importance of the concepts of left-definiteness and semi-left-definiteness. Its proof is elementary and hence omitted.

Theorem 1.47. If the Sturm-Liouville problem consisting of (0.1) and a selfadjoint boundary condition is left-definite or semi-left-definite, then its eigenvalues are all real.

Next, we recall some facts about the eigenvalues of (0.5). For every $A \in \mathcal{B}^{\mathbb{C}}$ and each $n \in \mathbb{N}_{0}:=\{0,1,2,3, \ldots\}$, let $\zeta_{n}(\boldsymbol{A})$ denote the $(n+1)$-th eigenvalue of the SLP consisting of (0.5) and $\boldsymbol{A}$. We also use the abbreviations $\zeta_{n}^{\boldsymbol{D}}=\zeta_{n}(\boldsymbol{D})$, etc. Let $\Psi(t, \zeta)=\left(\psi_{i j}(t, \zeta)\right)_{2 \times 2}$ be the principal matrix of $(0.5)$. Then,

$$
\Psi(b, 0)=\Phi(b, 0) .
$$

Lemma 1.49. If $\zeta_{0}^{D}>0$, then $\psi_{12}(b, 0)>0$; if $\zeta_{0}^{D}=0$, then $\psi_{12}(b, 0)=0$; in both cases, $\zeta_{n}(\boldsymbol{A})>0$ for all $\boldsymbol{A} \in \mathcal{B}^{\mathbb{C}}$ and $n \geq 2$.

Proof. By Lemma 2.1 in [3] and direct calculations, $\zeta \in \mathbb{R}$ is an eigenvalue for $\boldsymbol{D}$ if and only if $\psi_{12}(b, \zeta)=0$. Hence, if $\zeta_{0}^{D}=0$, then $\psi_{12}(b, 0)=0$. Moreover, by Theorem 3.1 in [1] or the Sturm Comparison Theorem, $\psi_{12}(b, \zeta)>0$ when $\zeta<\zeta_{0}^{D}$. Thus, if $\zeta_{0}^{D}>0$, then $\psi_{12}(b, 0)>0$.

In both cases, by Theorem 4.1 in [4],

$$
\zeta_{n}(\boldsymbol{A})>\zeta_{n-2}^{D} \geq 0 \quad \text { for } \boldsymbol{A} \in \mathcal{B}^{\mathbb{R}}, n \geq 2 .
$$

This completes the proof.

For any $\alpha \in(0, \pi]$ and $\beta \in[0, \pi)$, let

$$
\boldsymbol{S}_{\alpha, \beta}=\left[\begin{array}{cccc}
\cos \alpha & \sin \alpha & 0 & 0 \\
0 & 0 & \cos \beta & \sin \beta
\end{array}\right] .
$$

Then, the set $\mathcal{T}$ of separated selfadjoint BC's consists of these $\boldsymbol{S}_{\alpha, \beta}$ 's and is topologically a torus. Note that $\mathrm{SL}(2, \mathbb{R})$ also acts on $\mathcal{T}$. Moreover, $\boldsymbol{S}_{\alpha, \beta}$ can be defined for any $\alpha, \beta \in \mathbb{R}$, and sometimes normalizations of the ranges of $\alpha$ and $\beta$ different from the ones given just before (1.51) will be used. The following result is established in [2]. 
Lemma 1.52. For any $n \in \mathbb{N}_{0}$, as a function of $(\alpha, \beta), \zeta_{n}\left(\boldsymbol{S}_{\alpha, \beta}\right)$ is continuous on $(0, \pi] \times[0, \pi)$, strictly increasing in $\alpha$, and strictly decreasing in $\beta$. Moreover, for each $\alpha \in(0, \pi]$,

$$
\lim _{\beta \rightarrow \pi^{-}} \zeta_{0}\left(\boldsymbol{S}_{\alpha, \beta}\right)=-\infty, \quad \lim _{\beta \rightarrow \pi^{-}} \zeta_{n}\left(\boldsymbol{S}_{\alpha, \beta}\right)=\zeta_{n-1}\left(\boldsymbol{S}_{\alpha, 0}\right) \text { for } n \in \mathbb{N},
$$

and for each $\beta \in[0, \pi)$,

$$
\lim _{\alpha \rightarrow 0^{+}} \zeta_{0}\left(\boldsymbol{S}_{\alpha, \beta}\right)=-\infty, \quad \lim _{\alpha \rightarrow 0^{+}} \zeta_{n}\left(\boldsymbol{S}_{\alpha, \beta}\right)=\zeta_{n-1}\left(\boldsymbol{S}_{\pi, \beta}\right) \text { for } n \in \mathbb{N} .
$$

In order to describe the discontinuities of $\zeta_{n}$ on $\mathcal{B}^{\mathbb{R}}$, we let

$$
\begin{aligned}
& \mathcal{F}_{-}^{\mathbb{R}}=\left\{[K \mid-I] ; \quad K \in \mathrm{SL}(2, \mathbb{R}), k_{11} k_{12} \leq 0\right\}, \\
& \mathcal{G}_{-}^{\mathbb{R}}=\left\{\left[\begin{array}{cccc}
a_{1} & 1 & 0 & -r \\
r & 0 & -1 & b_{2}
\end{array}\right] ; \quad b_{2} \leq 0, a_{1}, r \in \mathbb{R}\right\}, \\
& \mathcal{H}_{-}^{\mathbb{R}}=\left\{\left[\begin{array}{cccc}
1 & a_{2} & -r & 0 \\
0 & r & b_{1} & -1
\end{array}\right] ; \quad a_{2} \leq 0, b_{1}, r \in \mathbb{R}\right\}, \\
& \mathcal{F}_{+}^{\mathbb{R}}=\mathcal{O}_{6}^{\mathbb{R}} \backslash \mathcal{F}_{-}^{\mathbb{R}}, \quad \mathcal{G}_{+}^{\mathbb{R}}=\mathcal{O}_{4}^{\mathbb{R}} \backslash \mathcal{G}_{-}^{\mathbb{R}}, \quad \mathcal{H}_{+}^{\mathbb{R}}=\mathcal{O}_{3}^{\mathbb{R}} \backslash \mathcal{H}_{-}^{\mathbb{R}}, \\
& \mathcal{I}_{-}^{\mathbb{R}}=\left\{\left[\begin{array}{cccc}
1 & a_{2} & 0 & r \\
0 & r & -1 & b_{2}
\end{array}\right] ; \quad a_{2}, b_{2} \leq 0, r \in \mathbb{R}, a_{2} b_{2} \geq r^{2}\right\} \text {, } \\
& \mathcal{I}_{+}^{\mathbb{R}}=\left\{\left[\begin{array}{cccc}
1 & a_{2} & 0 & r \\
0 & r & -1 & b_{2}
\end{array}\right] ; \quad a_{2}, b_{2}>0, r \in \mathbb{R}, a_{2} b_{2}>r^{2}\right\}, \\
& \mathcal{I}_{0}^{\mathbb{R}}=\mathcal{O}_{2}^{\mathbb{R}} \backslash\left(\mathcal{I}_{-}^{\mathbb{R}} \cup \mathcal{I}_{+}^{\mathbb{R}}\right), \\
& \mathcal{J}^{\mathbb{R}}=\left\{[K \mid-I] ; \quad K \in \mathrm{SL}(2, \mathbb{R}), k_{12}=0\right\} \\
& \cup\left\{\left[\begin{array}{cccc}
a_{1} & a_{2} & 0 & 0 \\
0 & 0 & b_{1} & b_{2}
\end{array}\right] \in \mathcal{B}^{\mathbb{R}} ; \quad a_{2} b_{2}=0\right\} .
\end{aligned}
$$

Note that the coupled BC's in $\mathcal{J}^{\mathbb{R}}$ are all in $\mathcal{F}_{-}^{\mathbb{R}}$, and

$$
\mathcal{J}^{\mathbb{R}} \cap \mathcal{T}=\left(\mathcal{J}^{\mathbb{R}} \cap \mathcal{G}_{-}^{\mathbb{R}}\right) \cup\left(\mathcal{J}^{\mathbb{R}} \cap \mathcal{H}_{-}^{\mathbb{R}}\right) \cup\{\boldsymbol{D}\} .
$$

The following is Theorem 3.39 in [4].

Theorem 1.64. The function $\zeta_{0}$ on $\mathcal{B}^{\mathbb{R}}$ is continuous on $\mathcal{B}^{\mathbb{R}} \backslash \mathcal{J}^{\mathbb{R}}$ and discontinuous at each point of $\mathcal{J}^{\mathbb{R}}$. For $n \in \mathbb{N}$, the function $\zeta_{n}$ is continuous on $\mathcal{B}^{\mathbb{R}} \backslash \mathcal{J}^{\mathbb{R}}$ and at each coupled boundary condition in $\mathcal{J}^{\mathbb{R}}$ where $\zeta_{n}=\zeta_{n-1}$, and is discontinuous at any other point of $\mathcal{J}^{\mathbb{R}}$. More precisely, for each coupled boundary condition $\boldsymbol{A} \in \mathcal{J}^{\mathbb{R}}$, the restriction of $\zeta_{n}$ to $\mathcal{F}_{-}^{\mathbb{R}}$ is continuous at $\boldsymbol{A}$ for $n \in \mathbb{N}_{0}$ and

$$
\lim _{\mathcal{F}_{+}^{\mathbb{R}} \ni \boldsymbol{B} \rightarrow \boldsymbol{A}} \zeta_{0}(\boldsymbol{B})=-\infty, \quad \lim _{\mathcal{F}_{+}^{\mathbb{R}} \ni \boldsymbol{B} \rightarrow \boldsymbol{A}} \zeta_{n}(\boldsymbol{B})=\zeta_{n-1}(\boldsymbol{A}) \text { for } n \in \mathbb{N} ;
$$

for each $\boldsymbol{A} \in \mathcal{J}^{\mathbb{R}} \cap \mathcal{G}_{-}^{\mathbb{R}}$, the restriction of $\zeta_{n}$ to $\mathcal{G}_{-}^{\mathbb{R}}$ is continuous at $\boldsymbol{A}$ for $n \in \mathbb{N}_{0}$, and

$$
\lim _{\mathcal{G}_{+}^{\mathbb{R}} \ni \boldsymbol{B} \rightarrow \boldsymbol{A}} \zeta_{0}(\boldsymbol{B})=-\infty, \quad \lim _{\mathcal{G}_{+}^{\mathbb{R}} \ni \boldsymbol{B} \rightarrow \boldsymbol{A}} \zeta_{n}(\boldsymbol{B})=\zeta_{n-1}(\boldsymbol{A}) \text { for } n \in \mathbb{N} ;
$$

for each $A \in \mathcal{J}^{\mathbb{R}} \cap \mathcal{H}_{-}^{\mathbb{R}}$, the restriction of $\zeta_{n}$ to $\mathcal{H}_{-}^{\mathbb{R}}$ is continuous at $\boldsymbol{A}$ for $n \in \mathbb{N}_{0}$, and

$$
\lim _{\mathcal{H}_{+}^{\mathbb{R}} \ni \boldsymbol{B} \rightarrow \boldsymbol{A}} \zeta_{0}(\boldsymbol{B})=-\infty, \quad \lim _{\mathcal{H}_{+}^{\mathbb{R}} \ni \boldsymbol{B} \rightarrow \boldsymbol{A}} \zeta_{n}(\boldsymbol{B})=\zeta_{n-1}(\boldsymbol{A}) \text { for } n \in \mathbb{N}
$$


while the restriction of $\zeta_{n}$ to $\mathcal{I}_{-}^{\mathbb{R}}$ is continuous at the Dirichlet boundary condition $D$ for $n \in \mathbb{N}_{0}$ and

$$
\begin{gathered}
\lim _{\mathcal{I}_{0}^{\mathbb{R}} \cup \mathcal{I}_{+}^{\mathbb{R}} \ni \boldsymbol{B} \rightarrow \boldsymbol{D}} \zeta_{0}(\boldsymbol{B})=\lim _{\mathcal{I}_{+}^{\mathbb{R}} \ni \boldsymbol{B} \rightarrow \boldsymbol{D}} \zeta_{1}(\boldsymbol{B})=-\infty, \\
\lim _{\mathcal{I}_{0}^{\mathbb{R}} \ni \boldsymbol{B} \rightarrow \boldsymbol{D}} \zeta_{n}(\boldsymbol{B})=\zeta_{n-1}(\boldsymbol{D}) \text { for } n \in \mathbb{N}, \\
\lim _{\mathcal{I}_{+}^{\mathbb{R}} \ni \boldsymbol{B} \rightarrow \boldsymbol{D}} \zeta_{n}(\boldsymbol{B})=\zeta_{n-2}(\boldsymbol{D}) \text { for } n \geq 2 .
\end{gathered}
$$

Remark 1.71. The real jump set $\mathcal{J}^{\mathbb{R}}$ does not depend on the SLE (0.5). It is easy to verify that $[A \mid B] \in \mathcal{B}^{\mathbb{R}}$ is in $\mathcal{J}^{\mathbb{R}}$ if and only if the $(2,1)$-entry in $B^{\mathrm{t}} A^{\mathrm{c}}$ is 0 , where $B^{\mathrm{t}}$ is the transpose of $B$ and $A^{\mathrm{c}}$ is the matrix of cofactors of $A$.

In order to describe the discontinuities of $\zeta_{n}$ as a function on $\mathcal{B}^{\mathbb{C}}$, we set

$$
\begin{aligned}
& \mathcal{F}_{-}^{\mathbb{C}}=\left\{\left[\mathrm{e}^{\mathrm{i} \theta} K \mid-I\right] ; \quad K \in \mathrm{SL}(2, \mathbb{R}), k_{11} k_{12} \leq 0, \theta \in[0, \pi)\right\}, \\
& \mathcal{G}_{-}^{\mathbb{C}}=\left\{\left[\begin{array}{cccc}
a_{1} & 1 & 0 & -\bar{z} \\
z & 0 & -1 & b_{2}
\end{array}\right] ; \quad b_{2} \leq 0, a_{1} \in \mathbb{R}, z \in \mathbb{C}\right\}, \\
& \mathcal{H}_{-}^{\mathbb{C}}=\left\{\left[\begin{array}{cccc}
1 & a_{2} & -\bar{z} & 0 \\
0 & z & b_{1} & -1
\end{array}\right] ; \quad a_{2} \leq 0, b_{1} \in \mathbb{R}, z \in \mathbb{C}\right\}, \\
& \mathcal{F}_{+}^{\mathbb{C}}=\mathcal{O}_{6}^{\mathbb{C}} \backslash \mathcal{F}_{-}^{\mathbb{C}}, \quad \mathcal{G}_{+}^{\mathbb{C}}=\mathcal{O}_{4}^{\mathbb{C}} \backslash \mathcal{G}_{-}^{\mathbb{C}}, \quad \mathcal{H}_{+}^{\mathbb{C}}=\mathcal{O}_{3}^{\mathbb{C}} \backslash \mathcal{H}_{-}^{\mathbb{C}}, \\
& \mathcal{I}_{-}^{\mathbb{C}}=\left\{\left[\begin{array}{cccc}
1 & a_{2} & 0 & \bar{z} \\
0 & z & -1 & b_{2}
\end{array}\right] ; \quad a_{2}, b_{2} \leq 0, z \in \mathbb{C}, a_{2} b_{2} \geq z \bar{z}\right\}, \\
& \mathcal{I}_{+}^{\mathbb{C}}=\left\{\left[\begin{array}{cccc}
1 & a_{2} & 0 & \bar{z} \\
0 & z & -1 & b_{2}
\end{array}\right] ; \quad a_{2}, b_{2}>0, z \in \mathbb{C}, a_{2} b_{2}>z \bar{z}\right\}, \\
& \mathcal{I}_{0}^{\mathbb{C}}=\mathcal{O}_{2}^{\mathbb{C}} \backslash\left(\mathcal{I}_{-}^{\mathbb{C}} \cup \mathcal{I}_{+}^{\mathbb{C}}\right), \\
& \mathcal{J}^{\mathbb{C}}=\left\{\left[\mathrm{e}^{\mathrm{i} \theta} K \mid-I\right] ; \quad K \in \mathrm{SL}(2, \mathbb{R}), k_{12}=0, \theta \in[0, \pi)\right\} \\
& \cup\left\{\left[\begin{array}{cccc}
a_{1} & a_{2} & 0 & 0 \\
0 & 0 & b_{1} & b_{2}
\end{array}\right] \in \mathcal{B}^{\mathbb{R}} ; \quad a_{2} b_{2}=0\right\} .
\end{aligned}
$$

Note that the separated BC's in $\mathcal{J}^{\mathbb{C}}$ other than the Dirichlet BC are in $\mathcal{G}_{-}^{\mathbb{C}} \cup \mathcal{H}_{-}^{\mathbb{C}}$. The following is Theorem 3.73 in [4].

Theorem 1.80. The conclusions of Theorem 1.64 still hold when the superscripts $\mathbb{R}$ in them are replaced by $\mathbb{C}$.

Remark 1.81. The jump set $\mathcal{J}^{\mathbb{C}}$ does not depend on the SLE (0.5). It is easy to verify that $[A \mid B] \in \mathcal{B}^{\mathbb{C}}$ is in $\mathcal{J}^{\mathbb{C}}$ if and only if the $(2,1)$-entry in $B^{\mathrm{t}} A^{\mathrm{c}}$ is 0 .

\section{$\S 2$. Separated Boundary CONDitions}

In this section, we determine the separated LD selfadjoint BC's. In addition to the notation $\boldsymbol{S}_{\alpha, \beta}$ defined in (1.51), we also use the abbreviation

$$
C=\left(\begin{array}{ll}
c_{11} & c_{12} \\
c_{21} & c_{22}
\end{array}\right):=\Phi(b, 0)=\Psi(b, 0) .
$$

Theorem 2.2. Let $\beta_{0}$ be the zero of $c_{12} \cos \beta+c_{22} \sin \beta$ on the interval $[0, \pi)$, and define a function $f:[0, \pi) \rightarrow \mathbb{R} \cup\{\infty\}$ by

$$
f(\beta)=\frac{c_{11} \cos \beta+c_{21} \sin \beta}{c_{12} \cos \beta+c_{22} \sin \beta} .
$$


i) If there is a left-definite selfadjoint boundary condition for (0.1), then the set of separated left-definite selfadjoint boundary conditions for (0.1) is

$$
\left\{\boldsymbol{S}_{\alpha, \beta} ; \quad 0 \leq \beta<\beta_{0}, \operatorname{arccot} f(\beta)<\alpha \leq \pi\right\},
$$

and the set of separated semi-left-definite selfadjoint boundary conditions for (0.1) is

$$
\left\{\boldsymbol{S}_{\alpha, \beta} ; \quad 0 \leq \beta \leq \beta_{0}, \operatorname{arccot} f(\beta) \leq \alpha \leq \pi\right\} .
$$

ii) If there is a semi-left-definite selfadjoint boundary condition for (0.1) and no left-definite selfadjoint boundary condition for (0.1), then the Dirichlet boundary condition is the only separated semi-left-definite selfadjoint boundary condition for (0.1).

Note that in (2.5) and the rest of this paper, we take $\operatorname{arccot} \infty=\pi$. This is related to the normalization of the ranges of $\alpha$ and $\beta$ just before (1.51).

Proof. i) By (4.32) in [3] and (2.1), the set of real selfadjoint BC's at which 0 is an eigenvalue of (0.5) equals

$$
\mathcal{S}_{0}^{\mathbb{R}}=\mathcal{S}^{\mathbb{R}} \bullet \Psi(b, 0)=\mathcal{S}^{\mathbb{R}} \bullet C,
$$

where $\mathcal{S}^{\mathbb{R}}=\left\{[A \mid B] \in \mathcal{B}^{\mathbb{R}} ; \operatorname{det}(A+B)=0\right\}$. Since a $\mathrm{BC}$ is separated after the action of $C$ if and only if it is separated, we have that

$$
\left(\mathcal{S}^{\mathbb{R}} \cdot C\right) \cap \mathcal{T}=\left(\mathcal{S}^{\mathbb{R}} \cap \mathcal{T}\right) \cdot C .
$$

Thus, the set of separated selfadjoint BC's at which 0 is an eigenvalue of (0.5) equals

$$
\mathcal{S}_{0}^{\mathbb{R}} \cap \mathcal{T}=\left(\mathcal{S}^{\mathbb{R}} \cap \mathcal{T}\right) \cdot C=\left\{\left[\begin{array}{cccc}
g(\beta) & h(\beta) & 0 & 0 \\
0 & 0 & \cos \beta & \sin \beta
\end{array}\right] ; \quad \beta \in[0, \pi)\right\},
$$

where $g(\beta)=c_{11} \cos \beta+c_{21} \sin \beta$ and $h(\beta)=c_{12} \cos \beta+c_{22} \sin \beta$. Our assumption and Proposition 1.46 imply that $\boldsymbol{D}$ is LD for (0.1). Then, by Theorem 1.44,

$$
\zeta_{0}^{\boldsymbol{D}}>0
$$

which together with Lemma 1.49 yields $c_{12}>0$ and hence $\beta_{0}>0$. The function $\operatorname{arccot} f(\beta)$ is continuous on $\left[0, \beta_{0}\right]$ and $\left(\beta_{0}, \pi\right)$. Actually, the curve on $\mathcal{T}$ described by

$$
\alpha=\operatorname{arccot} f(\beta):[0, \pi) \longrightarrow(0, \pi]
$$

is continuous, since it is the image of the diagonal circle on $\mathcal{T}$ under the action of $C$. Since $\zeta_{0}\left(\boldsymbol{S}_{\pi, 0}\right)=\zeta_{0}^{\boldsymbol{D}}>0$ and $\zeta_{0}\left(\boldsymbol{S}_{\pi, \beta}\right)$ is continuous in $\beta$ on $[0, \pi)$, we have that

$$
\zeta_{0}\left(\boldsymbol{S}_{\pi, \beta}\right)>0 \quad \text { for } \beta \in\left[0, \beta_{0}\right)
$$

and $\zeta_{0}\left(\boldsymbol{S}_{\pi, \beta_{0}}\right) \geq 0$. Since 0 is an eigenvalue of the SLP consisting of (0.5) and $\boldsymbol{S}_{\pi, \beta_{0}}$, we must have

$$
\zeta_{0}\left(\boldsymbol{S}_{\pi, \beta_{0}}\right)=0 .
$$

By Lemma 1.52, $\zeta_{0}\left(\boldsymbol{S}_{\pi, \beta}\right)$ is strictly decreasing in $\beta$ on $[0, \pi)$, from which and from (2.12) it follows that

$$
\zeta_{0}\left(\boldsymbol{S}_{\pi, \beta}\right)<0 \quad \text { for } \beta \in\left(\beta_{0}, \pi\right) .
$$


For each $\beta_{*} \in\left[0, \beta_{0}\right)$, let $\alpha_{*}=\operatorname{arccot} f\left(\beta_{*}\right)$. Then $\alpha_{*} \in(0, \pi)$, and similar arguments on $\alpha$ as above yield that

$$
\begin{aligned}
\zeta_{0}\left(\boldsymbol{S}_{\alpha, \beta_{*}}\right)<0 & \text { for } \alpha \in\left(0, \alpha_{*}\right), \\
\zeta_{0}\left(\boldsymbol{S}_{\alpha_{*}, \beta_{*}}\right) & =0, \\
\zeta_{0}\left(\boldsymbol{S}_{\alpha, \beta_{*}}\right)>0 & \text { for } \alpha \in\left(\alpha_{*}, \pi\right] .
\end{aligned}
$$

By Lemma 1.52 again, (2.12) and (2.13) imply that

$$
\zeta_{0}\left(\boldsymbol{S}_{\alpha, \beta}\right)<0 \quad \text { for } \alpha \in(0, \pi], \beta \in\left[\beta_{0}, \pi\right) .
$$

Hence, $\zeta_{0}\left(\boldsymbol{S}_{\alpha, \beta}\right)>0$ if and only if the inequalities in (2.4) hold, and $\zeta_{0}\left(\boldsymbol{S}_{\alpha, \beta}\right) \geq 0$ if and only if the inequalities in (2.5) are true. Therefore, by Theorem 1.44, the proof of i) is complete.

ii) Our assumption and Proposition 1.46 imply that $\boldsymbol{D}$ is SLD for (0.1) but not $\mathrm{LD}$ for (0.1). Then, from Theorem 1.44 it follows that $\zeta_{0}^{D}=0$, which together with Lemma 1.52 yields that $\zeta_{0}(\boldsymbol{A})<0$ for any $\boldsymbol{A} \in \mathcal{T} \backslash\{\boldsymbol{D}\}$. Thus, by Theorem 1.44 again, any separated selfadjoint BC other than $\boldsymbol{D}$ is not SLD for (0.1).

Remark 2.18. Our proof above plus some arguments along the same line can also show that when $D$ is LD for $(0.1), \zeta_{0}<0$ and $\zeta_{1}>0$ on the region between the two pieces of the curve in Figure 1. The upper piece of the curve in Figure 1 consists precisely of the separated selfadjoint BC's where $\left(\zeta_{0}<0\right.$ and) $\zeta_{1}=0$, and both $\zeta_{0}<0$ and $\zeta_{1}<0$ on the upper left region in Figure 1.

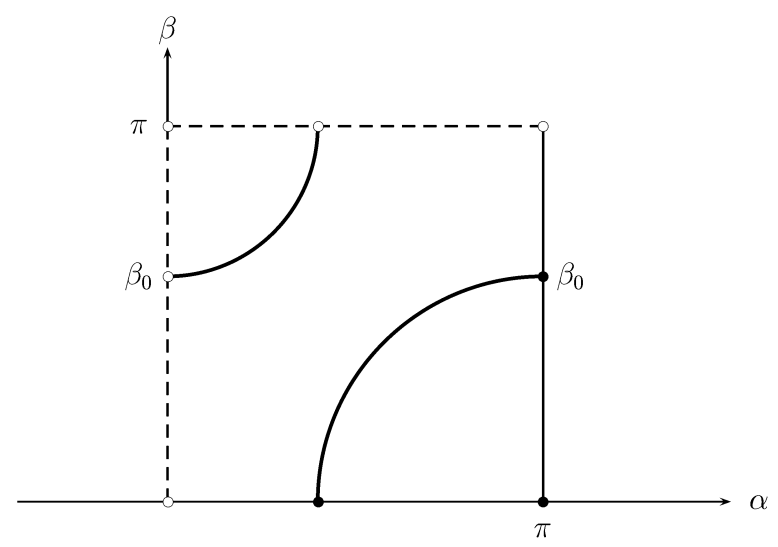

Figure 1. The Dirichlet boundary condition is left-definite.

When the SLP consisting of (0.1) and $\boldsymbol{D}$ is SLD but not LD, Figure 1 becomes Figure 2.

Figure 2, Remark 2.18 and the facts that

$$
\mathrm{DN}=S_{\pi, \pi / 2}, \quad \mathrm{ND}=\boldsymbol{S}_{\pi / 2,0}
$$

yield the following corollary, which can, of course, be shown directly using the main ideas in the above proof of Theorem 2.2. 


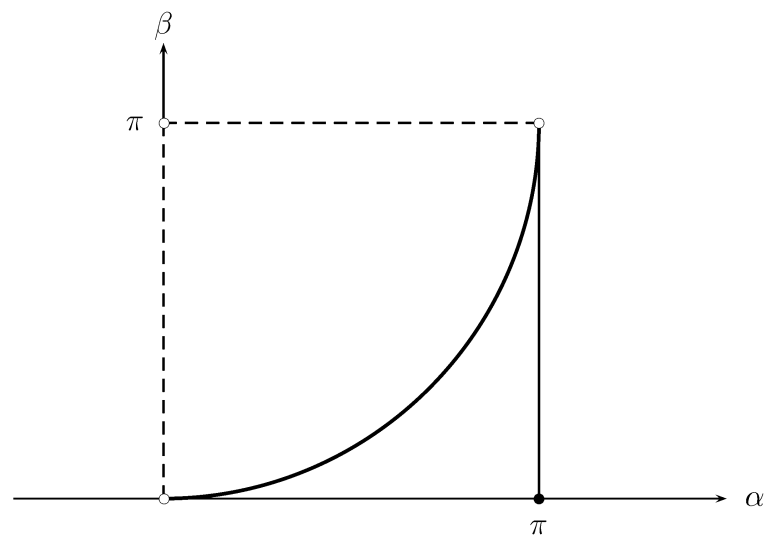

Figure 2. The Dirichlet boundary condition is semi-left-definite but not left-definite.

Corollary 2.20. If $\zeta_{0}^{D}=0$, then

$$
\begin{gathered}
\zeta_{0}^{\mathrm{DN}}<0, \quad \zeta_{1}^{\mathrm{DN}}>0, \quad \zeta_{0}^{\mathrm{ND}}<0, \quad \zeta_{1}^{\mathrm{ND}}>0, \\
\psi_{11}(b, 0)<0, \quad \psi_{22}(b, 0)<0 .
\end{gathered}
$$

To end this section, we give a class of examples and comment on the general case.

Example 2.22. Assume that $q \equiv 0$. Then, the fundamental matrix for (0.1) with $\lambda=0$ is

$$
\Phi(t, 0)=\left(\begin{array}{cc}
1 & \int_{a}^{t} \frac{\mathrm{d} s}{p(s)} \\
0 & 1
\end{array}\right),
$$

and hence

$$
C=\left(\begin{array}{ll}
1 & c \\
0 & 1
\end{array}\right), \text { where } c=\int_{a}^{b} \frac{\mathrm{d} t}{p(t)}>0
$$

Therefore,

$$
\beta_{0}=\operatorname{arccot} \frac{-1}{c} \in\left(\frac{\pi}{2}, \pi\right),
$$

the set of separated LD selfadjoint BC's for (0.1) is

$$
\left\{\boldsymbol{S}_{\alpha, \beta} ; \quad 0 \leq \beta<\beta_{0}, \operatorname{arccot} \frac{\cos \beta}{c \cos \beta+\sin \beta}<\alpha \leq \pi\right\},
$$

and the set of separated SLD selfadjoint BC's for (0.1) is

$$
\left\{S_{\alpha, \beta} ; \quad 0 \leq \beta \leq \beta_{0}, \operatorname{arccot} \frac{\cos \beta}{c \cos \beta+\sin \beta} \leq \alpha \leq \pi\right\} .
$$

In particular, these sets are non-empty.

Remark 2.28. In general, even though it is not possible to find the exact value of $C$, one can always approximate $C$ and hence the set (2.4) of separated LD selfadjoint BC's and the set (2.5) of separated SLD selfadjoint BC's. 


\section{§3. Real Boundary CONDitions}

In this section, we study the real (separated or coupled) LD selfadjoint BC's. As mentioned in the introduction, we need some results about the separated LD selfadjoint BC's in this study. Recall that the constant matrix $C$ is defined in (2.1).

Theorem 3.1. i) If there is a left-definite selfadjoint boundary condition for (0.1), then the space of real left-definite selfadjoint boundary conditions for (0.1) is

$$
\begin{aligned}
& \left\{\left[\begin{array}{cccc}
a_{1} c_{11}+c_{21} & a_{1} c_{12}+c_{22} & r & 0 \\
r c_{11} & r c_{12} & b_{1} & -1
\end{array}\right] ; \quad a_{1} \geq-\frac{c_{22}}{c_{12}}, r<-\frac{a_{1}+b_{1}}{2}\right\} \\
& \cup\left\{\boldsymbol{S}_{\alpha, 0} ; \quad \operatorname{arccot} \frac{c_{11}}{c_{12}}<\alpha \leq \pi\right\},
\end{aligned}
$$

and the space of real semi-left-definite selfadjoint boundary conditions for (0.1) is

$$
\begin{aligned}
& \left\{\left[\begin{array}{cccc}
a_{1} c_{11}+c_{21} & a_{1} c_{12}+c_{22} & r & 0 \\
r c_{11} & r c_{12} & b_{1} & -1
\end{array}\right] ; \quad a_{1} \geq-\frac{c_{22}}{c_{12}}, r \leq-\frac{a_{1}+b_{1}}{2}\right\} \\
& \cup\left\{S_{\alpha, 0} ; \quad \operatorname{arccot} \frac{c_{11}}{c_{12}} \leq \alpha \leq \pi\right\} \\
& \cup\left\{\left[\begin{array}{cccc}
c_{11} & c_{12} & -1 & 0 \\
c_{21} & c_{22} & d & -1
\end{array}\right] ; \quad d \leq 0\right\} .
\end{aligned}
$$

ii) If there is a semi-left-definite selfadjoint boundary condition for (0.1) and no left-definite selfadjoint boundary condition for (0.1), then $c_{11}<0, c_{12}=0, c_{22}=$ $1 / c_{11}<0$ and the space of real semi-left-definite selfadjoint boundary conditions for (0.1) is

$$
\{\boldsymbol{D}\} \cup\left\{\left[\begin{array}{cccc}
c_{11} & 0 & -1 & 0 \\
c_{21} & c_{22} & d & -1
\end{array}\right] ; \quad d \leq 0\right\} .
$$

Note that the first sets in (3.2) and (3.3) contain some separated BC's.

Proof. i) By our assumption, Proposition 1.46 and Theorem 1.44, we have that $\zeta_{0}^{D}>0$, which together with Lemma 1.49 yields that $c_{12}>0$. From Lemma 1.19 we see that

$$
\mathcal{B}^{\mathbb{R}}=\left(\mathcal{O}_{5}^{\mathbb{R}} \bullet C\right) \cup\left(\mathcal{K}^{\mathbb{R}} \cdot C\right) \cup\left(\mathcal{M}^{\mathbb{R}} \cdot C\right),
$$

which directs our analysis of $\zeta_{0}$ on $\mathcal{B}^{\mathbb{R}}$ to each of the 3 subsets on the right-hand side.

On the subset $\mathcal{O}_{5}^{\mathbb{R}} \bullet C$. Note that

$$
\mathcal{O}_{5}^{\mathbb{R}} \bullet C=\left\{\boldsymbol{A}\left(a_{1}, b_{1}, r\right) ; a_{1}, b_{1}, r \in \mathbb{R}\right\},
$$

where

$$
\boldsymbol{A}\left(a_{1}, b_{1}, r\right)=\left[\begin{array}{cccc}
a_{1} c_{11}+c_{21} & a_{1} c_{12}+c_{22} & r & 0 \\
r c_{11} & r c_{12} & b_{1} & -1
\end{array}\right] .
$$

By (4.32) and (4.31) in [3] and (2.1),

$$
\begin{aligned}
\mathcal{S}_{0}^{\mathbb{R}} \cap\left(\mathcal{O}_{5}^{\mathbb{R}} \bullet C\right) & =\left(\mathcal{S}^{\mathbb{R}} \cdot C\right) \cap\left(\mathcal{O}_{5}^{\mathbb{R}} \bullet C\right)=\left(\mathcal{S}^{\mathbb{R}} \cap \mathcal{O}_{5}^{\mathbb{R}}\right) \cdot C \\
& =\left\{\boldsymbol{A}\left(a_{1}, b_{1}, r\right) ; \quad a_{1}, b_{1}, r \in \mathbb{R}, a_{1}+b_{1}+2 r=0\right\},
\end{aligned}
$$

where $\mathcal{S}_{0}^{\mathbb{R}}$ is the set of real selfadjoint BC's at which 0 is an eigenvalue of $(0.5)$, and, by Remark 1.71,

$$
\mathcal{J}^{\mathbb{R}} \cap\left(\mathcal{O}_{5}^{\mathbb{R}} \bullet C\right)=\left\{\boldsymbol{A}\left(-c_{22} / c_{12}, b_{1}, r\right) ; b_{1}, r \in \mathbb{R}\right\} .
$$


These 2 planes will be denoted by $\mathcal{P}_{0}^{\mathbb{R}}$ and $\mathcal{P}_{\mathrm{J}}^{\mathbb{R}}$, respectively. They divide $\mathcal{O}_{5}^{\mathbb{R}} \bullet C \simeq$ $\mathbb{R}^{3}$ into 4 quarters. The continuity of $\zeta_{0}$ on $\mathcal{B}^{\mathbb{R}} \backslash \mathcal{J}^{\mathbb{R}}$ and the fact that $\zeta_{0} \neq 0$ on $\mathcal{B}^{\mathbb{R}} \backslash \mathcal{S}_{0}^{\mathbb{R}}$ imply that on each (open) quarter, $\zeta_{0}$ is either always positive or always negative.

Let $\epsilon>0$ be sufficiently small and consider

$$
A\left(\epsilon-\frac{c_{22}}{c_{12}}, \frac{1}{\epsilon}, 0\right)=\left[\begin{array}{cccc}
\epsilon c_{11} c_{12}-1 & \epsilon c_{12}^{2} & 0 & 0 \\
0 & 0 & -1 & \epsilon
\end{array}\right]
$$

which lies above $\mathcal{P}_{0}^{\mathbb{R}}$ (i.e., in the region with $r>-\frac{a_{1}+b_{1}}{2}$ ) and in front of $\mathcal{P}_{\mathrm{J}}^{\mathbb{R}}$ (i.e., in the region with $\left.a_{1}>-\frac{c_{22}}{c_{12}}\right)$. We note that

$$
\boldsymbol{A}\left(\epsilon-\frac{c_{22}}{c_{12}}, \frac{1}{\epsilon}, 0\right)=\boldsymbol{S}_{\alpha, \beta}
$$

for some $\alpha=\alpha(\epsilon) \in(0, \pi)$ and $\beta=\beta(\epsilon) \in(0, \pi)$ satisfying $\alpha \rightarrow \pi^{-}$and $\beta \rightarrow \pi^{-}$as $\epsilon \rightarrow 0^{+}$. By Lemma 1.52,

$$
\zeta_{0}\left(\boldsymbol{A}\left(\epsilon-\frac{c_{22}}{c_{12}}, \frac{1}{\epsilon}, 0\right)\right) \longrightarrow-\infty \quad \text { as } \epsilon \rightarrow 0^{+} .
$$

Thus, $\zeta_{0}$ is always negative on the upper front quarter (i.e., the quarter with $a_{1}>$ $-c_{22} / c_{12}$ and $\left.a_{1}+b_{1}+2 r>0\right)$. Alternatively, we consider the $\mathrm{BC}$

$$
\boldsymbol{A}\left(\epsilon-\frac{c_{22}}{c_{12}}, \frac{c_{22}}{c_{12}}-\frac{1}{\epsilon}, 0\right)=\left[\begin{array}{cccc}
\epsilon c_{11} c_{12}-1 & \epsilon c_{12}^{2} & 0 & 0 \\
0 & 0 & c_{12}-\epsilon c_{22} & \epsilon c_{12}
\end{array}\right],
$$

which lies below $\mathcal{P}_{0}^{\mathbb{R}}$ and in front of $\mathcal{P}_{\mathrm{J}}^{\mathbb{R}}$. As above,

$$
A\left(\epsilon-\frac{c_{22}}{c_{12}}, \frac{c_{22}}{c_{12}}-\frac{1}{\epsilon}, 0\right)=\boldsymbol{S}_{\hat{\alpha}, \hat{\beta}}
$$

for some $\hat{\alpha}=\hat{\alpha}(\epsilon) \in(0, \pi)$ and $\hat{\beta}=\hat{\beta}(\epsilon) \in(0, \pi)$ satisfying $\hat{\alpha} \rightarrow \pi^{-}$and $\hat{\beta} \rightarrow 0^{+}$as $\epsilon \rightarrow 0^{+}$. By Lemma 1.52 again,

$$
\zeta_{0}\left(A\left(\epsilon-\frac{c_{22}}{c_{12}}, \frac{c_{22}}{c_{12}}-\frac{1}{\epsilon}, 0\right)\right) \longrightarrow \zeta_{0}^{D}>0 \quad \text { as } \epsilon \rightarrow 0^{+} .
$$

Hence, $\zeta_{0}$ is always positive on the lower front quarter (i.e., the quarter with $a_{1}>$ $-c_{22} / c_{12}$ and $\left.a_{1}+b_{1}+2 r<0\right)$. Then, by the continuity of $\zeta_{0}$ away from $\mathcal{J}^{\mathbb{R}}, \zeta_{0}=0$ on the (open) front half of $\mathcal{P}_{0}^{\mathbb{R}}$ (i.e., the half with $a_{1}>-c_{22} / c_{12}$ ).

We observe that $\boldsymbol{D} \notin \mathcal{P}_{\mathrm{J}}^{\mathbb{R}}$. So, by Theorem $1.64, \zeta_{0}$ is continuous on the closure of one side of $\mathcal{P}_{\mathrm{J}}^{\mathbb{R}}$ and negative at the BC's in the remaining open side that are sufficiently close to $\mathcal{P} \mathbb{R}$. From what is proven above we then conclude that

$$
\zeta_{0} \text { is continuous on }\left\{\boldsymbol{A}\left(a_{1}, b_{1}, r\right) ; a_{1} \geq-c_{22} / c_{12}\right\}
$$

and $\zeta_{0}<0$ when $a_{1}<-c_{22} / c_{12}$ is sufficiently close to $-c_{22} / c_{12}$, and hence on each quarter behind $\mathcal{P}_{\mathrm{J}}^{\mathbb{R}}$. By the continuity of $\zeta_{0}$ away from $\mathcal{J}^{\mathbb{R}}$ again, $\zeta_{0} \leq 0$ on the back half of $\mathcal{P}_{0}^{\mathbb{R}}$ (i.e., the half with $a_{1}<-c_{22} / c_{12}$ ). Suppose that there exists a BC $\boldsymbol{B}_{0}$ in the back half of $\mathcal{P}_{0}^{\mathbb{R}}$ such that $\zeta_{0}\left(\boldsymbol{B}_{0}\right)=0$. Pick another BC $\boldsymbol{B}_{1} \in \mathcal{P}_{0}^{\mathbb{R}} \cap \mathcal{P}_{\mathrm{J}}^{\mathbb{R}}$ and let $\left\{\boldsymbol{B}_{s} ; 0 \leq s \leq 1\right\}$ be the segment from $\boldsymbol{B}_{0}$ to $\boldsymbol{B}_{1}$. By the fact that $\zeta_{0}\left(\boldsymbol{B}_{s}\right) \rightarrow-\infty$ as $s \rightarrow 1^{-}$and the continuity of $\zeta_{0}\left(\boldsymbol{B}_{s}\right)$ in $s$ on $[0,1)$, we may assume without loss of generality that $\zeta_{0}\left(\boldsymbol{B}_{s}\right)<0$ for $s \in(0,1)$. Since 0 is an eigenvalue of $(0.5)$ at each $\boldsymbol{B}_{s}$, Lemma 1.49 then implies that $\zeta_{1}\left(\boldsymbol{B}_{s}\right)=0$ for $s \in(0,1)$, and hence $\zeta_{1}\left(\boldsymbol{B}_{0}\right)=0$, i.e., 0 is a double eigenvalue of $(0.5)$ at $\boldsymbol{B}_{0}$. By Theorems 4.1 and 5.5 in [3],

$$
\boldsymbol{B}_{0}=[\Psi(b, 0) \mid-I]=[C \mid-I] \notin \mathcal{O}_{5}^{\mathbb{R}} \bullet C,
$$

which is impossible. Thus, $\zeta_{0}<0$ on the back half of $\mathcal{P}_{0}^{\mathbb{R}}$.

Therefore, $\zeta_{0}\left(\boldsymbol{A}\left(a_{1}, b_{1}, r\right)\right)>0$ if and only if $a_{1} \geq-c_{22} / c_{12}$ and $r<-\left(a_{1}+b_{1}\right) / 2$, while $\zeta_{0}\left(\boldsymbol{A}\left(a_{1}, b_{1}, r\right)\right) \geq 0$ if and only if $a_{1} \geq-c_{22} / c_{12}$ and $r \leq-\left(a_{1}+b_{1}\right) / 2$. 
$\underline{\text { On the subset }} \mathcal{K}^{\mathbb{R}} \cdot C$. We note that

$$
\mathcal{K}^{\mathbb{R}} \cdot C=\left\{\boldsymbol{E}\left(a_{2}, b_{2}, r\right) ; \quad a_{2}, b_{2}, r \in \mathbb{R}, a_{2} b_{2}=r^{2}\right\},
$$

where

$$
\boldsymbol{E}\left(a_{2}, b_{2}, r\right)=\left[\begin{array}{cccc}
c_{11}+a_{2} c_{21} & c_{12}+a_{2} c_{22} & 0 & r \\
r c_{21} & r c_{22} & -1 & b_{2}
\end{array}\right] .
$$

Then, as in the previous case,

$$
\begin{aligned}
\mathcal{S}_{0}^{\mathbb{R}} \cap\left(\mathcal{K}^{\mathbb{R}} \bullet C\right) & =\left\{\boldsymbol{E}\left(a_{2}, a_{2},-a_{2}\right) ; a_{2} \in \mathbb{R}\right\}, \\
\mathcal{J}^{\mathbb{R}} \cap\left(\mathcal{K}^{\mathbb{R}} \bullet C\right) & =\left\{\boldsymbol{E}\left(a_{2}, 0,0\right) ; a_{2} \in \mathbb{R}\right\} .
\end{aligned}
$$

Consider a fixed BC $\boldsymbol{E}\left(a_{2}, b_{2}, r\right) \in \mathcal{K}^{\mathbb{R}} \bullet C$.

If $b_{2}=0$, then $r=0$, and the $\mathrm{BC}$ is

$$
\boldsymbol{E}\left(a_{2}, 0,0\right)=\left[\begin{array}{cccc}
c_{11}+a_{2} c_{21} & c_{12}+a_{2} c_{22} & 0 & 0 \\
0 & 0 & -1 & 0
\end{array}\right]=\boldsymbol{S}_{\alpha, 0}
$$

for some $\alpha=\alpha\left(a_{2}\right) \in(0, \pi]$. Hence, by Theorem 2.2, this BC is LD for (0.1) if and only if $\operatorname{arccot} \frac{c_{11}}{c_{12}}<\alpha \leq \pi$, and it is SLD for (0.1) if and only if $\operatorname{arccot} \frac{c_{11}}{c_{12}} \leq$ $\alpha \leq \pi$. Observe that all the $\alpha$ 's in $(0, \pi]$, except for the one $\alpha_{*}$ determined by $\left(c_{21}^{2}+c_{22}^{2}\right)\left(\cos \alpha_{*}, \sin \alpha_{*}\right)= \pm\left(c_{21}, c_{22}\right)$, are used in (3.22).

Before going on to the remaining subcase, we pause to mention that for $r \neq 0$ and $s>1$,

$$
\boldsymbol{E}\left(a_{2} s, b_{2} s, r\right)=\boldsymbol{A}(\tilde{a}, \tilde{b}, \tilde{r}) \in \mathcal{O}_{5}^{\mathbb{R}} \bullet C
$$

where

$$
\tilde{a}=\frac{b_{2} s}{r^{2} s^{2}-r^{2}}, \quad \tilde{b}=\frac{a_{2} s}{r^{2} s^{2}-r^{2}}, \quad \tilde{r}=\frac{r}{r^{2} s^{2}-r^{2}},
$$

and

$$
\boldsymbol{E}\left(a_{2} s, b_{2} s, r\right) \longrightarrow \boldsymbol{E}\left(a_{2}, b_{2}, r\right) \quad \text { as } s \rightarrow 1^{+} .
$$

If $b_{2}>0$ and $r \neq 0$, then we have that $\tilde{a}>-c_{22} / c_{12}$ and $\tilde{a}+\tilde{b}+2 \tilde{r}>0$ for $s>1$ and sufficiently close to 1 ; if $b_{2}<0$ and $r \neq 0$, then $\tilde{a}<-c_{22} / c_{12}$ for $s>1$ and sufficiently close to 1 . What we have proven in the previous case implies that when $b_{2} \neq 0$ and $r \neq 0$,

$$
\zeta_{0}(\boldsymbol{A}(\tilde{a}, \tilde{b}, \tilde{r}))<0
$$

for $s>1$ and sufficiently close to 1 . Thus, from the continuity of $\zeta_{0}$ on $\mathcal{B}^{\mathbb{R}} \backslash \mathcal{J}^{\mathbb{R}}$ we conclude that if $b_{2} \neq 0$, then

$$
\zeta_{0}\left(\boldsymbol{E}\left(a_{2}, b_{2}, r\right)\right) \leq 0 .
$$

To see when the equality holds, we only need to focus our attention further to the BC's $\boldsymbol{E}\left(a_{2}, a_{2},-a_{2}\right)$ in $\mathcal{S}_{0}^{\mathbb{R}} \cap\left(\mathcal{K}^{\mathbb{R}} \bullet C\right)$ with $a_{2} \neq 0$. We note that when $a_{2}$ is sufficiently close to 0 ,

$$
\boldsymbol{E}\left(a_{2}, a_{2},-a_{2}\right)=\left[\begin{array}{cccc}
\left(c_{11}+a_{2} c_{21}\right) h & 1 & 0 & -a_{2} h \\
a_{2} h & 0 & -1 & a_{2} c_{12} h
\end{array}\right],
$$

where $h=1 /\left(c_{12}+a_{2} c_{22}\right)$. Thus, when $a_{2} \leq 0$ is sufficiently close to 0 ,

$$
\boldsymbol{E}\left(a_{2}, a_{2},-a_{2}\right) \in \mathcal{G}_{-}^{\mathbb{R}} .
$$


So, by Theorem 1.64, $\zeta_{0}\left(\boldsymbol{E}\left(a_{2}, a_{2},-a_{2}\right)\right)$ and $\zeta_{1}\left(\boldsymbol{E}\left(a_{2}, a_{2},-a_{2}\right)\right)$ are continuous in $a_{2}$ on $(-\epsilon, 0]$ for some $\epsilon>0$, and hence on $(-\infty, 0]$. Since

$$
\boldsymbol{E}(0,0,0)=\left[\begin{array}{cccc}
c_{11} & c_{12} & 0 & 0 \\
0 & 0 & -1 & 0
\end{array}\right]
$$

from Theorem 2.2 we know that $\zeta_{0}(\boldsymbol{E}(0,0,0))=0$ and $\zeta_{1}(\boldsymbol{E}(0,0,0))>0$. The fact that 0 cannot be a double eigenvalue of $(0.5)$ at $\boldsymbol{E}\left(a_{2}, a_{2},-a_{2}\right)$ then implies that for $a_{2} \in(-\infty, 0)$,

$$
\zeta_{0}\left(\boldsymbol{E}\left(a_{2}, a_{2},-a_{2}\right)\right)=0, \quad \zeta_{1}\left(\boldsymbol{E}\left(a_{2}, a_{2},-a_{2}\right)\right)>0 .
$$

Moreover, when $a_{2}>0$ is sufficiently close to 0 ,

$$
\boldsymbol{E}\left(a_{2}, a_{2},-a_{2}\right) \in \mathcal{G}_{+}^{\mathbb{R}} .
$$

By Theorem 1.64 again and Lemma 1.49,

$$
\zeta_{0}\left(\boldsymbol{E}\left(a_{2}, a_{2},-a_{2}\right)\right)<0, \quad \zeta_{1}\left(\boldsymbol{E}\left(a_{2}, a_{2},-a_{2}\right)\right)=0
$$

for $a_{2}>0$ sufficiently close to 0 , and hence for all $a_{2} \in(0,+\infty)$. Therefore, for $\boldsymbol{E}\left(a_{2}, b_{2}, r\right) \in \mathcal{K}^{\mathbb{R}} \bullet C$ with $b_{2} \neq 0$,

$$
\zeta_{0}\left(\boldsymbol{E}\left(a_{2}, b_{2}, r\right)\right) \leq 0,
$$

with equality holding only when $a_{2} \in(-\infty, 0), b_{2}=a_{2}$ and $r=-a_{2}$, i.e., only when

$$
\boldsymbol{E}\left(a_{2}, b_{2}, r\right)=\left[\begin{array}{cccc}
c_{11} & c_{12} & -1 & 0 \\
c_{21} & c_{22} & 1 / a_{2} & -1
\end{array}\right] \quad \text { with } a_{2}<0 .
$$

On the subset $\mathcal{M}^{\mathbb{R}} \bullet C$. As in the previous two cases, direct calculations yield

$$
\begin{aligned}
& \mathcal{S}_{0}^{\mathbb{R}} \cap\left(\mathcal{M}^{\mathbb{R}} \bullet C\right)=\{[C \mid-I]\}, \\
& \mathcal{J}^{\mathbb{R}} \cap\left(\mathcal{M}^{\mathbb{R}} \cdot C\right)=\{\mathrm{ND} \cdot C\}=\left\{\left[\begin{array}{cccc}
c_{21} & c_{22} & 0 & 0 \\
0 & 0 & -1 & 0
\end{array}\right]\right\} .
\end{aligned}
$$

Note that ND. $C=\boldsymbol{S}_{\alpha, 0}$ for some $\alpha \in(0, \pi]$ and, by Theorem 2.2, it needs to satisfy $\operatorname{arccot} \frac{c_{11}}{c_{12}}<\alpha \leq \pi$ for left-definiteness and $\operatorname{arccot} \frac{c_{11}}{c_{12}} \leq \alpha \leq \pi$ for semi-leftdefiniteness.

The subset $\left(\mathcal{M}^{\mathbb{R}} \backslash\{\mathrm{ND}\}\right) \bullet C$ is part of the limit points of $\mathcal{K}^{\mathbb{R}} \bullet C$ by Lemma 1.19, and hence consists of limit points of

$$
\left(\mathcal{K}^{\mathbb{R}} \cdot C\right) \backslash\left\{\boldsymbol{E}\left(a_{2}, 0,0\right) ; a_{2} \in \mathbb{R}\right\} .
$$

So, from the continuity of $\zeta_{0}$ at the points of $\left(\mathcal{M}^{\mathbb{R}} \backslash\{\mathrm{ND}\}\right) \bullet C$ and the results of the previous case, $\zeta_{0} \leq 0$ on $\left(\mathcal{M}^{\mathbb{R}} \backslash\{\mathrm{ND}\}\right) \bullet C$. Moreover, by (3.35), or by Theorems 4.1 and 5.5 in [3] and Lemma 1.49 above,

$$
\zeta_{0}([C \mid-I])=0 .
$$

Therefore, $\zeta_{0} \leq 0$ on $\left(\mathcal{M}^{\mathbb{R}} \backslash\{\mathrm{ND}\}\right) \cdot C$, and equality holds only at $[C \mid-I]$.

ii) By our assumption, Proposition 1.46 and Theorem 1.44, $\zeta_{0}^{D}=0$. Then, Lemma 1.49 and Corollary 2.20 imply that $c_{11}<0, c_{12}=0$ and $c_{22}<0$. Therefore, our conclusion follows from i) by taking the limit $c_{12} \rightarrow 0^{+}$, which can be obtained by a perturbation of the SLE (0.1). 
To end this section, we give the following continuation of Example 2.22.

Example 3.40. Assume that $q \equiv 0$. Then, by Example 2.22,

$$
C=\left(\begin{array}{ll}
1 & c \\
0 & 1
\end{array}\right), \text { where } c=\int_{a}^{b} \frac{\mathrm{d} t}{p(t)}>0
$$

Therefore, the set of real LD selfadjoint BC's for (0.1) is

$$
\begin{aligned}
& \left\{\left[\begin{array}{cccc}
a_{1} & a_{1} c+1 & r & 0 \\
r & r c & b_{1} & -1
\end{array}\right] ; \quad a_{1} \geq-\frac{1}{c}, r<-\frac{a_{1}+b_{1}}{2}\right\} \\
& \cup\left\{\boldsymbol{S}_{\alpha, 0} ; \quad \operatorname{arccot} \frac{1}{c}<\alpha \leq \pi\right\},
\end{aligned}
$$

and the set of real SLD selfadjoint BC's for (0.1) is

$$
\begin{aligned}
& \left\{\left[\begin{array}{cccc}
a_{1} & a_{1} c+1 & r & 0 \\
r & r c & b_{1} & -1
\end{array}\right] ; \quad a_{1} \geq-\frac{1}{c}, r \leq-\frac{a_{1}+b_{1}}{2}\right\} \\
& \cup\left\{\boldsymbol{S}_{\alpha, 0} ; \quad \operatorname{arccot} \frac{1}{c} \leq \alpha \leq \pi\right\} \cup\left\{\left[\begin{array}{cccc}
1 & c & -1 & 0 \\
0 & 1 & d & -1
\end{array}\right] ; \quad d \leq 0\right\} .
\end{aligned}
$$

In particular, these sets are non-empty.

There is a remark similar to Remark 2.28 about the set (3.2) of real LD selfadjoint BC's and the set (3.3) of real SLD selfadjoint BC's in general. We omit the details.

\section{$\S 4$. Complex Boundary CONDitions}

In this section, we present the results in the most generality, i.e., determine all LD selfadjoint BC's. The proofs here are very similar to those in the previous section, and hence are omitted. Recall again that the constant matrix $C$ is defined in $(2.1)$.

Theorem 4.1. i) If the space $\mathcal{B}_{\mathrm{L}}^{\mathbb{C}}$ of left-definite selfadjoint boundary conditions for (0.1) is non-empty, then

$$
\begin{aligned}
\mathcal{B}_{\mathrm{L}}^{\mathbb{C}}= & \left\{\left[\begin{array}{cccc}
a_{1} c_{11}+c_{21} & a_{1} c_{12}+c_{22} & \bar{z} & 0 \\
z c_{11} & z c_{12} & b_{1} & -1
\end{array}\right] ; \quad \begin{array}{c}
a_{1} \geq-c_{22} / c_{12} \\
\operatorname{Re} z<-\left(a_{1}+b_{1}\right) / 2
\end{array}\right\} \\
& \cup\left\{\boldsymbol{S}_{\alpha, 0} ; \quad \operatorname{arccot} \frac{c_{11}}{c_{12}}<\alpha \leq \pi\right\},
\end{aligned}
$$

and the space $\mathcal{B}_{\mathrm{SL}}^{\mathbb{C}}$ of semi-left-definite selfadjoint boundary conditions for (0.1) is

$$
\begin{aligned}
& \left\{\left[\begin{array}{cccc}
a_{1} c_{11}+c_{21} & a_{1} c_{12}+c_{22} & \bar{z} & 0 \\
z c_{11} & z c_{12} & b_{1} & -1
\end{array}\right] ; \quad \begin{array}{c}
a_{1} \geq-c_{22} / c_{12} \\
\cup \operatorname{Re} z \leq-\left(a_{1}+b_{1}\right) / 2
\end{array}\right\} \\
& \cup\left\{\boldsymbol{S}_{\alpha, 0} ; \quad \operatorname{arccot} \frac{c_{11}}{c_{12}} \leq \alpha \leq \pi\right\} \\
& \cup\left\{\left[\begin{array}{cccc}
c_{11} & c_{12} & -1 & 0 \\
c_{21} & c_{22} & d & -1
\end{array}\right] ; \quad d \leq 0\right\} .
\end{aligned}
$$

ii) If $\mathcal{B}_{\mathrm{L}}^{\mathbb{C}}$ is empty and $\mathcal{B}_{\mathrm{SL}}^{\mathbb{C}}$ is not, then $c_{11}<0, c_{12}=0, c_{22}=1 / c_{11}<0$ and

$$
\mathcal{B}_{\mathrm{L}}^{\mathbb{C}}=\{\boldsymbol{D}\} \cup\left\{\left[\begin{array}{cccc}
c_{11} & 0 & -1 & 0 \\
c_{21} & c_{22} & d & -1
\end{array}\right] ; \quad d \leq 0\right\} .
$$

Note that the first sets in the right-hand side of (4.2) and (4.3) contain some separated BC's. The following is a by-product of Theorem 4.1, part ii), and was mentioned in the introduction. 
Corollary 4.5. For a given regular Sturm-Liouville equation with a positive leading coefficient and a definite weight function, the collection of selfadjoint boundary conditions whose first eigenvalue equals the first Dirichlet eigenvalue $r_{0}$ is

$$
\{\boldsymbol{D}\} \cup\left\{\left[\begin{array}{cccc}
\omega_{11}\left(b, r_{0}\right) & 0 & -1 & 0 \\
\omega_{21}\left(b, r_{0}\right) & \omega_{22}\left(b, r_{0}\right) & d & -1
\end{array}\right] ; d \leq 0\right\},
$$

where $\Omega=\left(\omega_{i j}\right)_{2 \times 2}$ is the principal matrix of the given Sturm-Liouville equation.

Proof. Without loss of generality, we assume that the given SLE is (0.5). If $\zeta_{0}^{D}=0$, then our claim is just Theorem 4.1, part ii). In general, we consider the SLE

$$
-\left(p y^{\prime}\right)^{\prime}+\left(q-\zeta_{0}^{D}|w|\right) y=\tilde{\zeta}|w| y \text { on }(a, b),
$$

whose first Dirichlet eigenvalue $\tilde{\zeta}_{0}^{D}=0$. Thus, the collection of selfadjoint BC's at which the first eigenvalue of (4.7) equals 0 , i.e., the collection of selfadjoint BC's at which the first eigenvalue of $(0.5)$ equals $\zeta_{0}^{D}$, is

$$
\{\boldsymbol{D}\} \cup\left\{\left[\begin{array}{cccc}
\tilde{c}_{11} & \tilde{c}_{12} & -1 & 0 \\
\tilde{c}_{21} & \tilde{c}_{22} & d & -1
\end{array}\right] ; d \leq 0\right\}
$$

where $\widetilde{C}=\widetilde{\Psi}(b, 0)$ with $\widetilde{\Psi}$ being the principal matrix of (4.7). Since

$$
\widetilde{\Psi}(t, \tilde{\zeta})=\Psi\left(t, \tilde{\zeta}+\zeta_{0}^{\boldsymbol{D}}\right)
$$

for $t \in[a, b]$ and $\tilde{\zeta} \in \mathbb{R}$, the collection of selfadjoint BC's at which the first eigenvalue of $(0.5)$ equals $\zeta_{0}^{D}$ is

$$
\{\boldsymbol{D}\} \cup\left\{\left[\begin{array}{llcc}
\psi_{11}\left(b, \zeta_{0}^{\boldsymbol{D}}\right) & \psi_{12}\left(b, \zeta_{0}^{\boldsymbol{D}}\right) & -1 & 0 \\
\psi_{21}\left(b, \zeta_{0}^{\boldsymbol{D}}\right) & \psi_{22}\left(b, \zeta_{0}^{\boldsymbol{D}}\right) & d & -1
\end{array}\right] ; d \leq 0\right\} .
$$

By Lemma 1.49, $\tilde{\psi}_{12}(b, 0)=0$. Hence, $\psi_{12}\left(b, \zeta_{0}^{D}\right)=0$. This completes the proof.

Remark 4.11. Let $T_{0}$ denote the minimal operator of the SLE (0.5) in the weighted Hilbert space $\mathrm{L}^{2}((a, b), \mathbb{C} ;|w|)$. Among all the selfadjoint extensions of $T_{0}$, the one most frequently used in applied mathematics and mathematical physics is the so-called Friedrichs extension $S_{\mathrm{F}}$. Friedrichs' celebrated construction of $S_{\mathrm{F}}$ does not explicitly refer to any $\mathrm{BC}$, but it is known [7] that the $\mathrm{BC}$ corresponding to this extension in the regular case considered here is the Dirichlet BC $\boldsymbol{D}$. An important property of $S_{\mathrm{F}}$ is that it has the same lower bound as $T_{0}$. However, this property does not determine $S_{\mathrm{F}}$ uniquely, i.e., in general there are other selfadjoint extensions with the same lower bound as $T_{0}$. Since this lower bound is the first Dirichlet eigenvalue $r_{0}$, we note that (4.6) characterizes all selfadjoint extensions of $T_{0}$ which have the same lower bound as $T_{0}$.

We make some additional observations from (4.6).

i) For no separated selfadjoint BC other than $\boldsymbol{D}$ does the corresponding extension preserve the lower bound of $T_{0}$.

ii) For no non-real coupled selfadjoint $\mathrm{BC}$ does the corresponding extension preserve the lower bound of $T_{0}$.

Thus, only extensions corresponding to some real coupled selfadjoint BC's preserve the lower bound of $T_{0}$, and all those BC's are given by (4.6).

To end this section, we have the following continuation of Examples 2.22 and 3.40 . 
Example 4.12. Assume that $q \equiv 0$. Then, by Example 2.22,

$$
C=\left(\begin{array}{ll}
1 & c \\
0 & 1
\end{array}\right), \text { where } c=\int_{a}^{b} \frac{\mathrm{d} t}{p(t)}>0
$$

Therefore, the set of LD selfadjoint BC's for (0.1) is

$$
\begin{aligned}
& \left\{\left[\begin{array}{cccc}
a_{1} & a_{1} c+1 & \bar{z} & 0 \\
z & z c & b_{1} & -1
\end{array}\right] ; \quad a_{1} \geq-\frac{1}{c}, \operatorname{Re} z<-\frac{a_{1}+b_{1}}{2}\right\} \\
& \cup\left\{\boldsymbol{S}_{\alpha, 0} ; \quad \operatorname{arccot} \frac{1}{c}<\alpha \leq \pi\right\},
\end{aligned}
$$

and the set of SLD selfadjoint BC's for (0.1) is

$$
\begin{aligned}
& \left\{\left[\begin{array}{cccc}
a_{1} & a_{1} c+1 & \bar{z} & 0 \\
z & z c & b_{1} & -1
\end{array}\right] ; \quad a_{1} \geq-\frac{1}{c}, \operatorname{Re} z \leq-\frac{a_{1}+b_{1}}{2}\right\} \\
& \cup\left\{\boldsymbol{S}_{\alpha, 0} ; \quad \operatorname{arccot} \frac{1}{c} \leq \alpha \leq \pi\right\} \cup\left\{\left[\begin{array}{cccc}
1 & c & -1 & 0 \\
0 & 1 & d & -1
\end{array}\right] ; \quad d \leq 0\right\} .
\end{aligned}
$$

In particular, these sets are non-empty.

There is a remark similar to Remark 2.28 about the set (4.2) of LD selfadjoint BC's and the set (4.3) of SLD selfadjoint BC's in general. We omit the details.

\section{§5. Geometric shape}

In this section, we determine the shape of the spaces of the real and complex selfadjoint BC's for left-definiteness and semi-left-definiteness.

By the half-open two-vertex cone solid in $\mathbb{R}^{3}$ we will mean the set

$$
\left\{(x, y, z) \in \mathbb{R}^{3} ; x^{2}+y^{2}<1, x^{2}+y^{2} \leq z<2-x^{2}-y^{2}\right\},
$$

and by the closed two-vertex cone solid in $\mathbb{R}^{3}$ the set

$$
\left\{(x, y, z) \in \mathbb{R}^{3} ; x^{2}+y^{2} \leq 1, x^{2}+y^{2} \leq z \leq 2-x^{2}-y^{2}\right\} .
$$

Theorem 5.3. If the space of real left-definite selfadjoint boundary conditions for (0.1) is non-empty, then it is diffeomorphic to the half-open two-vertex cone solid in $\mathbb{R}^{3}$, and the space of real semi-left-definite selfadjoint boundary conditions for (0.1) is diffeomorphic to the closed two-vertex cone solid in $\mathbb{R}^{3}$.

Proof. We continue to use the notation introduced in the proof of Theorem 3.1, and we set $\alpha_{0}=\operatorname{arccot} \frac{c_{11}}{c_{12}}$. So,

$$
\begin{aligned}
& \left\{\boldsymbol{A}\left(-\frac{c_{22}}{c_{12}}, b_{1}, r\right) ; b_{1} \in \mathbb{R}, r<\frac{c_{22}}{2 c_{12}}-\frac{b_{1}}{2}\right\} \\
& \quad \cup\left\{\boldsymbol{A}\left(a_{1}, b_{1},-\frac{a_{1}+b_{1}}{2}\right) ; a_{1} \geq-\frac{c_{22}}{c_{12}}, b_{1} \in \mathbb{R}\right\}
\end{aligned}
$$

is part of the boundary $\partial \mathcal{B}_{\mathrm{L}}^{\mathbb{R}}$ of the space $\mathcal{B}_{\mathrm{L}}^{\mathbb{R}}$ of real LD selfadjoint BC's for (0.1), while

$$
\left\{\boldsymbol{A}\left(a_{1}, b_{1}, r\right) ; a_{1}>-\frac{c_{22}}{c_{12}}, r<-\frac{a_{1}+b_{1}}{2}\right\}
$$

consists of interior points of $\mathcal{B}_{\mathrm{L}}^{\mathbb{R}}$. If $\boldsymbol{P}_{\infty}$ is in the closure of $\mathcal{B}_{\mathrm{L}}^{\mathbb{R}}$, then there is a sequence $\left\{\boldsymbol{P}_{n}\right\}_{n \geq 1}$ of points in $\mathcal{B}_{\mathrm{L}}^{\mathbb{R}}$ that converges to $\boldsymbol{P}_{\infty}$. The description of the discontinuities of $\zeta_{0}$ on $\mathcal{B}_{\mathrm{S}}^{\mathbb{R}}$ in Theorem 1.64 and the fact that $\zeta_{0}\left(\boldsymbol{P}_{n}\right)>0$ for $n \geq 1$ imply that the sequence $\left\{\boldsymbol{P}_{n}\right\}_{n \geq 1}$ approaches $\boldsymbol{P}_{\infty}$ from the continuity side of $\boldsymbol{P}_{\infty}$, and hence $\zeta_{0}\left(\boldsymbol{P}_{\infty}\right) \geq 0$, i.e., $\boldsymbol{P}_{\infty}$ belongs to the space $\mathcal{B}_{\mathrm{SL}}^{\mathbb{R}}$ of real SLD selfadjoint 
BC's for (0.1). So, we have shown that the closure of $\mathcal{B}_{\mathrm{L}}^{\mathbb{R}}$ is contained in $\mathcal{B}_{\mathrm{SL}}^{\mathbb{R}}$. Note that

$$
\boldsymbol{A}\left(-\frac{c_{22}}{c_{12}}, b_{1}, r\right)=\left[\begin{array}{cccc}
-1 & 0 & c_{12} r & 0 \\
c_{11} r & c_{12} r & b_{1} & -1
\end{array}\right]
$$

Hence,

$$
\lim _{b_{1} \rightarrow-\infty} \boldsymbol{A}\left(-\frac{c_{22}}{c_{12}}, b_{1}, 0\right)=\boldsymbol{D}=\boldsymbol{S}_{\pi, 0} .
$$

When $\gamma=: b_{1}+c_{11} c_{12} r^{2} \neq 0$,

$$
\boldsymbol{A}\left(-\frac{c_{22}}{c_{12}}, b_{1}, r\right)=\left[\begin{array}{cccc}
1 & c_{12}^{2} r^{2} / \gamma & 0 & -c_{12} r / \gamma \\
0 & -c_{12} r / \gamma & -1 & 1 / \gamma
\end{array}\right]
$$

So, for $\tau<0$,

$$
\begin{aligned}
\lim _{r \rightarrow \pm \infty} & A\left(-\frac{c_{22}}{c_{12}}, \tau c_{12} r^{2}, r\right) \\
= & \lim _{r \rightarrow \pm \infty}\left[\begin{array}{cccc}
c_{11}+\tau & c_{12} & 0 & -1 / r \\
0 & -1 / r & -c_{11}-\tau & 1 /\left(c_{12} r^{2}\right)
\end{array}\right] \\
= & {\left[\begin{array}{cccc}
c_{11}+\tau & c_{12} & 0 & 0 \\
0 & 0 & -1 & 0
\end{array}\right]=S_{\alpha, 0}, }
\end{aligned}
$$

where $\alpha=\operatorname{arccot} \frac{c_{11}+\tau}{c_{12}} \in\left(\alpha_{0}, \pi\right)$. Since $\tau<0$ is arbitrary, the above limit can be any point in the set $\left\{\boldsymbol{S}_{\alpha, 0} ; \alpha_{0}<\alpha \leq \pi\right\}$. Hence,

$$
\mathcal{L}:=\left\{\boldsymbol{A}\left(-\frac{c_{22}}{c_{12}}, b_{1}, r\right) ; b_{1} \in \mathbb{R}, r<\frac{c_{22}}{2 c_{12}}-\frac{b_{1}}{2}\right\} \cup\left\{\boldsymbol{S}_{\alpha, 0} ; \alpha_{0}<\alpha \leq \pi\right\}
$$

is topologically an open disk in $\mathcal{J}^{\mathbb{R}}$. Since $\partial \mathcal{B}_{\mathrm{L}}^{\mathbb{R}}$ is closed, we have that $\mathcal{L} \subseteq \partial \mathcal{B}_{\mathrm{L}}^{\mathbb{R}}$.

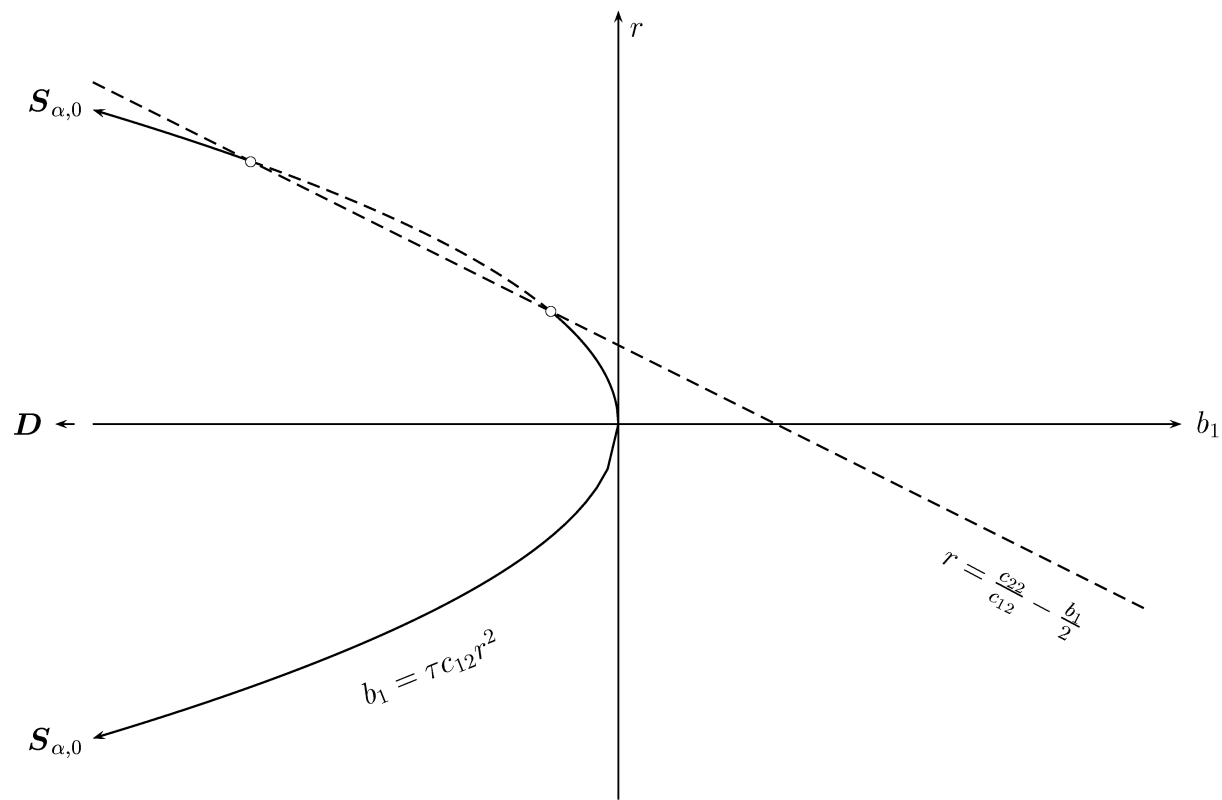

Figure 3. Separated part of the boundary as limit of the coupled part of the boundary. 
Moreover, the limit

$$
\begin{aligned}
\lim _{b_{1} \rightarrow \pm \infty} & A\left(-\frac{c_{22}}{c_{12}}, b_{1}, \frac{c_{22}}{2 c_{12}}-\frac{b_{1}}{2}\right) \\
= & \lim _{b_{1} \rightarrow \pm \infty}\left[\begin{array}{cccc}
c_{11} & c_{12} & 2 b_{1} c_{12} / \delta & -2 c_{12} / \delta \\
2 / \delta & 0 & -1 & 0
\end{array}\right] \\
= & {\left[\begin{array}{cccc}
c_{11} & c_{12} & 0 & 0 \\
0 & 0 & -1 & 0
\end{array}\right]=S_{\alpha_{0}, 0}, }
\end{aligned}
$$

where $\delta=c_{22}-b_{1} c_{12}$, and two more limits similar to (5.7) and (5.9) together yield that

$$
\begin{aligned}
\mathcal{U}:= & \left\{\boldsymbol{A}\left(a_{1}, b_{1},-\frac{a_{1}+b_{1}}{2}\right) ; a_{1} \geq-\frac{c_{22}}{c_{12}}, b_{1} \in \mathbb{R}\right\} \\
& \cup\left\{\boldsymbol{S}_{\alpha_{0}, 0}\right\} \cup\left\{\left[\begin{array}{cccc}
c_{11} & c_{12} & -1 & 0 \\
c_{21} & c_{22} & d & -1
\end{array}\right] ; d \leq 0\right\} \\
\subseteq & \partial \mathcal{B}_{\mathrm{L}}^{\mathbb{R}}
\end{aligned}
$$

and is topologically a closed disk in $\mathcal{S}_{0}^{\mathbb{R}}$. Hence, $\partial \mathcal{B}_{\mathrm{L}}^{\mathbb{R}}=\mathcal{L} \cup \mathcal{U}$. Since

$$
\mathcal{W}:=\left\{A\left(-\frac{c_{22}}{c_{12}}, b_{1}, \frac{c_{22}}{2 c_{12}}-\frac{b_{1}}{2}\right) ; b_{1} \in \mathbb{R}\right\} \cup\left\{\boldsymbol{S}_{\alpha_{0}, 0}\right\}
$$

is a circle, $\mathcal{B}_{\mathrm{L}}^{\mathbb{R}}$ is homeomorphic to the half-open two-vertex cone solid in $\mathbb{R}^{3}$, and $\mathcal{B}_{\mathrm{SL}}^{\mathbb{R}}$ to the closed two-vertex cone solid in $\mathbb{R}^{3}$.

It is shown in [3] that $\mathcal{S}_{0}^{\mathbb{R}}$ is a collapsed torus with $[C \mid-I] \in \mathcal{U}$ as its only singular point. Similarly one can show that $\mathcal{J}^{\mathbb{R}}$ is a collapsed torus with $\boldsymbol{D} \in \mathcal{L}$ as its only singular point. Away from $\boldsymbol{S}_{\alpha_{0}, 0}$, the curve $\mathcal{W}$ is smooth. Near $\boldsymbol{S}_{\alpha_{0}, 0}=\boldsymbol{D} \boldsymbol{\bullet} C, \mathcal{S}_{0}^{\mathbb{R}}$ is the surface

$$
b_{2}=-a_{2}-2 s
$$

in

$$
\mathcal{O}_{2}^{\mathbb{R}} \bullet C=\left\{\left[\begin{array}{cccc}
1 & a_{2} & 0 & s \\
0 & s & -1 & b_{2}
\end{array}\right] \bullet C ; a_{2}, b_{2}, s \in \mathbb{R}\right\}
$$

while $\mathcal{J}^{\mathbb{R}}$ is defined by

$$
b_{2}=c_{22} s^{2} /\left(c_{12}+a_{2} c_{22}\right) .
$$

From (5.14) and (5.16) we see that $\mathcal{S}_{0}^{\mathbb{R}}$ and $\mathcal{J}^{\mathbb{R}}$ are transversal at $\boldsymbol{S}_{\alpha_{0}, 0}$, i.e., the point in $\mathcal{O}_{2}^{\mathbb{R}} \bullet C$ with $\left(a_{2}, b_{2}, s\right)=(0,0,0)$. Hence, as part of their intersection, $\mathcal{W}$ is also smooth at $\boldsymbol{S}_{\alpha_{0}, 0}$. Therefore, $\mathcal{B}_{\mathrm{L}}^{\mathbb{R}}$ is diffeomorphic to the half-open two-vertex cone solid in $\mathbb{R}^{3}$, and $\mathcal{B}_{\mathrm{SL}}^{\mathbb{R}}$ to the closed two-vertex cone solid in $\mathbb{R}^{3}$.

We use the graphs in Figure 4 to illustrate the shapes of the space $\mathcal{B}_{\mathrm{L}}^{\mathbb{R}}$ of real LD selfadjoint BC's for (0.1) and the space $\mathcal{B}_{\mathrm{SL}}^{\mathbb{R}}$ of real SLD selfadjoint BC's for (0.1).

Remark 5.17. The part of $\mathcal{B}_{\mathrm{L}}^{\mathbb{R}}$ that is near $\boldsymbol{D}$ can be viewed very clearly in $\mathcal{O}_{2}^{\mathbb{R}}$ : it is exactly $\mathcal{I}_{-}^{\mathbb{R}}$ with part of its big end cut off (by the zero set of $\zeta_{0}$ ).

Similarly to the real case, by the half-open two-vertex cone solid in $\mathbb{R}^{4}$ we will mean the set

$$
\left\{(w, x, y, z) \in \mathbb{R}^{4} ; w^{2}+x^{2}+y^{2}<1, w^{2}+x^{2}+y^{2} \leq z<2-w^{2}-x^{2}-y^{2}\right\}
$$



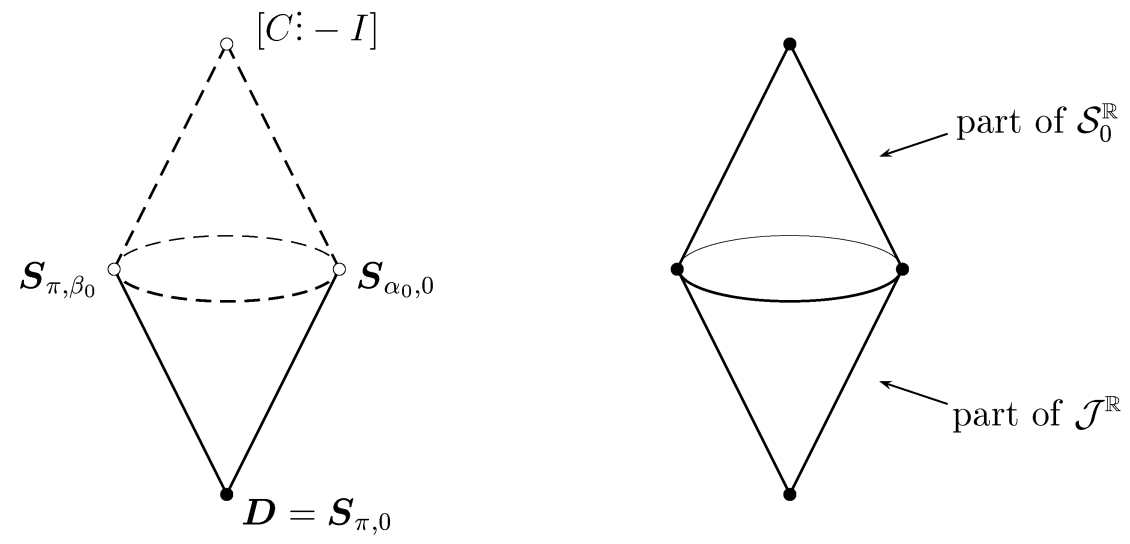

Figure 4 . The Dirichlet boundary condition is left-definite.

and by the closed two-vertex cone solid in $\mathbb{R}^{4}$ the set

$$
\left\{(w, x, y, z) \in \mathbb{R}^{4} ; w^{2}+x^{2}+y^{2} \leq 1, w^{2}+x^{2}+y^{2} \leq z \leq 2-w^{2}-x^{2}-y^{2}\right\} .
$$

Moreover, we have the following result, whose proof is very similar that of Theorem 5.3 and hence is omitted.

Theorem 5.20. If the space of left-definite selfadjoint boundary conditions for (0.1) is non-empty, then it is diffeomorphic to the half-open two-vertex cone solid in $\mathbb{R}^{4}$, and the space of semi-left-definite selfadjoint boundary conditions for (0.1) is diffeomorphic to the closed two-vertex cone solid in $\mathbb{R}^{4}$.

Finally, we turn to the case where the space of LD selfadjoint BC's is empty and the space of SLD selfadjoint BC's is not.

Theorem 5.21. When $\mathcal{B}_{\mathrm{L}}^{\mathbb{C}}$ is empty and $\mathcal{B}_{\mathrm{SL}}^{\mathbb{C}}$ is not, i.e., when $\mathcal{B}_{\mathrm{L}}^{\mathbb{R}}$ is empty and $\mathcal{B}_{\mathrm{SL}}^{\mathbb{R}}$ is not, $\mathcal{B}_{\mathrm{SL}}^{\mathbb{C}}=\mathcal{B}_{\mathrm{SL}}^{\mathbb{R}}$ is a simple smooth curve joining the Dirichlet boundary condition $D$ to the unique boundary condition $[C \mid-I]$ at which 0 is a double eigenvalue of (0.1).

Proof. The curve is simple by (3.4) or (4.4). Also, it is smooth away from the end point $D$. Since $c_{11}<0$ in this case, we have that for any sufficiently large $\delta>0$,

$$
\begin{aligned}
& \{\boldsymbol{D}\} \cup\left\{\left[\begin{array}{cccc}
c_{11} & 0 & -1 & 0 \\
c_{21} & c_{22} & d & -1
\end{array}\right] ; \quad d<-\delta\right\} \\
& =\left\{\left[\begin{array}{cccc}
1 & c_{22} r & 0 & -r \\
0 & -r & -1 & c_{11} r
\end{array}\right] ; \quad 0 \leq r<\frac{1}{c_{21}-c_{11} \delta}\right\},
\end{aligned}
$$

i.e., the curve is also smooth at $\boldsymbol{D}$. By Theorems 4.1 and 5.5 in $[3],[C \mid-I]$ is the unique $\mathrm{BC}$ at which 0 is a double eigenvalue of (0.1).

\section{ACKNOWLEDGMENT}

This work is supported by the National Science Foundation through the grant DMS-9973108. We would like to thank Eric Behr for drawing the figures in this paper. 


\section{REFERENCES}

1. M. Eastham, Q. Kong, H. Wu and A. Zettl: Inequalities among eigenvalues of Sturm-Liouville problems. J. Inequalities and Appl. 3 (1999), 25-43. MR 2000k:34132

2. W. Everitt, M. Möller and A.Zettl: Discontinuous dependence of the n-th Sturm-Liouville eigenvalue. "General Inequalities" ed. C. Bandle, W. Everitt, L. Losonszi and W. Walter, 145150. Birkhäuser, 1997. MR 98f:34033

3. Q. Kong, H. Wu and A. Zettl: Geometric aspects of Sturm-Liouville problems, I. Structures on spaces of boundary conditions. Proc. Royal Soc. Edinburgh 130A (2000), 561-589. MR 2001e:34042

4. Q. Kong, H. Wu and A. Zettl: Dependence of the $n$-th Sturm-Liouville eigenvalue on the problem. J. Differential Equations 156 (1999), 328-354. MR 2000k:34045

5. Q. Kong, H. Wu and A. Zettl: Left-definite Sturm-Liouville problems. J. Differential Equations 177 (2001), 1-26.

6. A. Mingarelli: A survey of the regular weighted Sturm-Liouville problem-the non-definite case. "International Workshop on Applied Differential Equations", 109-137. World Scientific, Singapore, 1986. MR 88e:34005

7. H. Niessen and A.Zettl: Singular Sturm-Liouville problems: the Friedrichs extension and comparison of eigenvalues. Proc. London Math. Soc. 64 (1992), 545-578. MR 93e:47060

Department of Mathematics, Northern Illinois University, DeKalb, Illinois 60115

Department of Mathematics, Northern Illinois University, DeKalb, Illinois 60115

Department of Mathematics, Northern Illinois University, DeKalb, Illinois 60115

Department of Mathematics, Northern Illinois University, DeKalb, Illinois 60115 